\title{
Modal identification of linear non-proportionally damped systems by wavelet transform
}

\author{
Silvano Erlicher* , Pierre Argoul \\ Laboratoire d'Analyse des Matériaux et Identification LAMI \\ (ENPC/LCPC-Institut Navier), 6 et 8 av. B. Pascal, Cité Descartes, \\ Champs-sur-Marne, 77455 Marne-la-Vallée, Cedex 2, France
}

\begin{abstract}
A time-frequency identification technique based on wavelet transform is formulated and applied to free-decay responses of linear systems with non-proportional viscous damping. The Cauchy mother wavelet is used. Frequencies, modal damping ratios and complex mode shapes are identified from output-only free vibration signals. This identification technique has also shown to be effective when the (non-proportional) damping is significant.
\end{abstract}

Key words: Modal identification, Complex modes, Shock response, Time-frequency analysis, Wavelet transform, Cauchy mother wavelet PACS:

* Tel: +33 1641537 80, Fax: +33164153741

Email address: erlicher@lami.enpc.fr (Silvano Erlicher). 


\section{Introduction}

Damping in mechanical systems is related to the energy dissipation and derives from several physical phenomena, such as, for instance, friction at micro and macroscopic levels. Its mathematical modeling is non-trivial and remains an active area of research in structural dynamics. Because of the underlying complexity when representing damping, linearity, time-invariance and causality are usually assumed for the sake of simplicity. Under that assumption, in spatially-discretized Multi-Degree of Freedom (MDoF) systems the dissipation force can be defined as a convolution integral between the velocity and a suitable positive and decreasing time-function called the memory kernel. This case is sometimes referred to as general linear damping (see e.g. [1-3]). As a result, the dissipation force in the present state depends on the present and past values of the velocity. The memory kernel could be further assumed to be the Dirac delta distribution, which leads to the case of linear viscous damping, where the dissipation force depends only on the present value of the velocity. Moreover, the value of the modal damping ratio is classically assumed to be strictly less than one for each mode (the damping coefficient is less than the critical damping, e.g. see [4]), which is the mathematical condition for underdamped systems.

Vibratory motion exists only in underdamped systems.

Rayleigh [5] made one more assumption by taking the viscous damping forces to be proportional to the inertia and stiffness forces. Since introduction of this model, known as Rayleigh damping model, has been extensively used for modeling structural behavior because it leads to a rather simple mathematical treatment. It is a special case of a more general model $[6,7]$, also referred to as proportional damping, in which the normal modes are real and the modal damping matrix is diagonal [8]. The choice of proportional damping is justified in numerous practical situations since the influence of the off-diagonal terms is negligible when damping is light and the different modal frequencies are well separated. However, there is no mathematical theory or sufficient experimental evidence as to why damping in a physical system should be described by proportional damping. In fact, experimental analysis shows that for most real life structures, the criterion for proportional viscous damping is no longer satisfied, as these structures possess complex modes instead of real normal modes (see e.g. [9]). A natural question is then how to define an index of non-proportionality characterizing the level of non-proportionality of the modal damping for each mode. The index presented in this paper was first introduced by Adhikari [10] on the basis of a specific normalization of the complex mode shapes. Other indices have also been proposed in the literature (see [10] and the references cited there). It is precisely the modal identification of non-proportionally viscous damped systems that is the focus of this paper. 
Modal identification can be usually performed either by time-domain or frequency-domain techniques (for an exhaustive review, the reader is referred to [11]). More recently, time-frequency domain techniques have been proposed for identification purposes. Among them, the Continuous Wavelet Transform (CWT) has been extensively studied in the past ten years, with application to both linear and nonlinear systems [12-16]. Different mother wavelets, in particular the Cauchy mother wavelet, have been used and compared, in order to identify modal frequencies and modal damping ratios [15]. The effect of noise in the data as well as the treatment of edge effects of the CWT and the choice of the optimal mother wavelet are reviewed in [15-18]. The identification by CWT of real mode shapes is also discussed in [15]. In all these contributions damping is assumed to be proportional and very small, with the exception of [18], where the damping is assumed to be non-proportional, but very small. The main topic of this paper is the modal analysis of a system having non-proportional and non-small damping, using the CWT with the Cauchy mother wavelet on after-shock output signals.

Section 2 reviews the main concepts concerning linear systems having non-proportional viscous damping. In particular, the definition of a suited index of non-proportionality is discussed. Section 3 recalls the definition of the CWT as well as the main properties of signals useful for the computation of the CWT. In addition, the Cauchy mother wavelet is presented in detail. Section 4 is devoted to the presentation of the CWT-based identification technique. Some aspects of edge effects are analyzed. In Section 5, the proposed identification technique is applied to the free-decay responses of two 4-DoFs systems whose modes have relatively high value of non-proportionality index. Noise and noise-free signals are considered. Moreover, relatively significant values of the damping ratio (around 35\%) are also introduced to test the efficiency of the technique. The values of the identified frequencies, damping ratios and complex mode shapes are calculated and compared to the theoretical ones. Section 6 deals with the application of the proposed modal identification technique to the free-decay responses of a plexiglass plate. Finally, some concluding remarks are given in the last section.

\section{$2 \quad$ Linear systems with viscous damping}

Consider a MDoF linear system with $N$ degrees of freedom. Let $\mathbf{u}=\left[u_{1}, \ldots, u_{N}\right]^{T}$ be the column vector of the nodal generalized displacements and let $\mathbf{F}(t)$ be the external force vector. The dissipation force vector is denoted $\mathbf{F}_{d}(t)$. When $\mathbf{F}_{d}(t)=\mathbf{C} \dot{\mathbf{u}}(t)$, the (linear) damping is usually referred to as viscous: note that in this case no past value of the velocity appears in $\mathbf{F}_{d}(t)$. The superposed dot indicates the derivative with respect 
to time and $\mathbf{C}$ is the so-called viscous damping matrix. Conversely, a definition where $\mathbf{F}_{d}(t)$ depends on the history of the past of $\dot{\mathbf{u}}(t)$ via convolution integrals with suitable kernel functions leads to the general linear damping model. In this paper, only viscous damping is considered and the system dynamic equation reads

$$
\left\{\begin{array}{l}
\mathbf{M} \ddot{\mathbf{u}}(t)+\mathbf{C} \dot{\mathbf{u}}(t)+\mathbf{K u}(t)=\mathbf{F}(t) \\
\mathbf{u}(0)=\mathbf{u}_{0} \\
\dot{\mathbf{u}}(0)=\mathbf{v}_{0}
\end{array}\right.
$$

where $\mathbf{u}_{0}$ and $\mathbf{v}_{0}$ are the initial values of the displacement and velocity vectors respectively and $\mathbf{M}, \mathbf{C}$ and $\mathbf{K}$ are $N \times N$ symmetric real matrices. It is assumed that all of the generalized displacements $u_{k}$ are associated with nodes where the concentrated mass is non-zero. Hence, the mass matrix $\mathbf{M}$ is definite positive. Moreover, the stiffness matrix $\mathbf{K}$ and the viscous damping matrix $\mathbf{C}$ are assumed to be at least nonnegative definite.

\subsection{Eigenvalues and vectors of non-proportionally damped systems}

For systems with general viscous damping, the modal analysis can be conveniently made by converting (1) into a first order matrix equation:

$$
\mathcal{A} \dot{\mathbf{y}}+\mathcal{B} \mathbf{y}=\mathbf{0}
$$

where

$$
\mathcal{A}=\left(\begin{array}{cc}
\mathrm{C} & \mathrm{M} \\
\mathrm{M} & 0
\end{array}\right) \quad \mathcal{B}=\left(\begin{array}{cc}
\mathrm{K} & 0 \\
0 & -\mathrm{M}
\end{array}\right)
$$

are real symmetric matrices of dimension $2 N$ and

$$
\mathbf{y}=\left(\begin{array}{c}
\mathbf{u} \\
\dot{\mathbf{u}}
\end{array}\right)
$$

is the state vector. By introducing the trial solution

$$
\mathbf{y}=\boldsymbol{\psi} e^{s t}
$$


in the system (2), the following equation is easily derived:

$$
(s \mathcal{A}+\mathcal{B}) \boldsymbol{\psi}=\mathbf{0}
$$

where $s$ is the characteristic value (or eigenvalue) which is solution of the characteristic polynomial $\operatorname{det}(s \mathcal{A}+\mathcal{B})=0$, and $\boldsymbol{\psi}$ is the associated complex eigenvector having $2 N$ components. By using (3), one can see that (5) is equivalent to

$$
\psi=\left(\begin{array}{c}
\phi \\
s \phi
\end{array}\right)
$$

and

$$
\left(s^{2} \mathbf{M}+s \mathbf{C}+\mathbf{K}\right) \boldsymbol{\phi}=\mathbf{0}
$$

where $\phi$ is a complex eigenvector of $N$ elements, which is also sometimes referred to as the latent vector [19]. Let us note that Eq. (5) has $2 N$ complex vector solutions $\boldsymbol{\psi}_{j}$ of $2 N$ components and Eq. (7) has $2 N$ complex vector solutions $\phi_{j}$ of $N$ components. The eigenvalues $s_{j}$ of Eq. (7) fulfil the following equation (see Appendix A.1.1 for details)

$$
s_{j}^{2}+2 \xi_{j} \omega_{j} s_{j}+\omega_{j}^{2}=0
$$

where $\omega_{j}$ is the $\mathrm{j}$-th modal circular frequency and $\xi_{j}$ is the $\mathrm{j}$-th modal damping ratio. The elements of $\mathbf{C}$ are assumed not to be too large so that all $\xi_{j}$ are less than 1 (underdamped system) and the eigenvalues $s_{j}$, solutions of (8), occur in complex conjugate pairs

$$
s_{j}=-\xi_{j} \omega_{j} \pm i \omega_{j} \sqrt{1-\xi_{j}^{2}}
$$

where

$$
\omega_{j}=\left|s_{j}\right| \quad \text { and } \quad \xi_{j}=\frac{-\operatorname{Re}\left(s_{j}\right)}{\left|s_{j}\right|}
$$

The same property holds for the corresponding eigenvectors $\boldsymbol{\phi}_{j}$ and $\boldsymbol{\psi}_{j}$. When the so-called proportional or classical damping condition holds [6]

$$
\left(\mathbf{M}^{-1} \mathbf{C}\right)\left(\mathbf{M}^{-1} \mathbf{K}\right)=\left(\mathbf{M}^{-1} \mathbf{K}\right)\left(\mathbf{M}^{-1} \mathbf{C}\right)
$$


the imaginary part of all eigenvectors is zero, i.e. the standard situation of real normal modes is retrieved (see Appendix A.1.3 for details).

\subsection{An index of non-proportionality of complex mode shapes}

Let $\boldsymbol{\varphi}_{j}, \boldsymbol{\phi}_{j} \in \mathbb{C}^{N}$ be two complex vectors. Then, the vector

$$
\phi_{j, o p t}=\frac{\boldsymbol{\varphi}_{j}^{T} \bar{\phi}_{j}}{\left\|\phi_{j}\right\|^{2}} \phi_{j}
$$

is the orthogonal projection of $\boldsymbol{\varphi}_{j}$ on $\phi_{j}$ (see Figure 1), where

$\left\|\phi_{j}\right\|=\sqrt{\phi_{j}^{T} \bar{\phi}_{j}}$. One can note that, given $\boldsymbol{\varphi}_{j}$, the vector $\phi_{j, o p t}$ remains unchanged for every initial normalization of $\phi_{j}$, i.e. if $\phi_{j}^{\prime}=c_{j} \phi_{j}$ with $c_{j} \in \mathbb{C}$, then

$$
\phi_{j, o p t}^{\prime}=\bar{c}_{j} \frac{\boldsymbol{\varphi}_{j}^{T} \bar{\phi}_{j}}{\left|c_{j}\right|^{2} \|\left.\phi_{j}\right|^{2}} c_{j} \phi_{j}=\phi_{j, o p t}
$$

Moreover, the inequalities $\left\|\boldsymbol{\phi}_{j, \text { opt }}\right\| \leq\left\|\boldsymbol{\varphi}_{j}\right\|$ and $\left\|\boldsymbol{\varphi}_{j}-\boldsymbol{\phi}_{j, \text { opt }}\right\| \leq\left\|\boldsymbol{\varphi}_{j}\right\|$ hold by Pitagora's theorem. The angle $\alpha_{j}$ between $\boldsymbol{\varphi}_{j}$ and $\phi_{j, \text { opt }}$ always belongs to the interval $[0, \pi / 2]$ (Figure 1 ) and its sine can be adopted as a measure of the degree of parallelism between the two vectors:

$$
I_{n p}^{(j)}=\frac{\left\|\phi_{j, o p t}-\boldsymbol{\varphi}_{j}\right\|}{\left\|\boldsymbol{\varphi}_{j}\right\|}=\sin \left(\alpha_{j}\right)
$$

This index is equal to zero when the two vectors are parallel and equal to one when they are orthogonal in $\mathbb{C}^{N}$. Moreover, $I_{n p}^{(j)}$ does not change when $\boldsymbol{\varphi}_{j}$ is multiplied by some real or complex normalization scalar.

If $\phi_{j}$ represents the j-th complex mode of a given system with non-proportional damping and $\boldsymbol{\varphi}_{j} \in \mathbb{R}^{N}$ is the corresponding real normal mode of the associated undamped system, then $\phi_{j, \text { opt }}$ can be interpreted as an optimally normalized complex mode, while the positive scalar $I_{n p}^{(j)} \in[0,1]$ can be adopted as an index of the non-proportionality of the damping, independent of the chosen normalization of both the complex and the real modes. This quantity is associated with each single mode shape and was first interpreted as a non-proportionality index by Adhikari [10]. If the real mode shape $\boldsymbol{\varphi}_{j}$ is not known, another index $\tilde{I}_{n p}^{(j)}$ has to be found. One alternative definition can be postulated by introducing the real part $R e\left(\phi_{j}\right)$ of $\phi_{j}$ 
instead of $\boldsymbol{\varphi}_{j}$ in Eqs. (12) and (13). The index obtained by this procedure is a measure of the importance of the imaginary part of $\phi_{j}$ with respect to the real one. The numerical examples of the last section illustrate that $\tilde{I}_{n p}^{(j)}$ is an acceptable approximation of $I_{n p}^{(j)}$.

\subsection{Free vibration of non-proportionally damped systems}

Due to the linearity of the non-proportionally damped system (1), the free-decay response $\mathbf{u}(t)=\left[u_{1}(t), \ldots, u_{k}(t), \ldots, u_{N}(t)\right]^{T}$ can be written as the sum of $N$ modal solutions:

$$
u_{k}(t)=\sum_{j=1}^{N} u_{k j}(t)=\sum_{j=1}^{N} \frac{1}{2}\left(G_{j} \phi_{k j} e^{s_{j} t}+\bar{G}_{j} \bar{\phi}_{k j} e^{\bar{s}_{j} t}\right) \in \mathbb{R}
$$

where $u_{k j}(t)$ is the $j-t h$ frequency component of $u_{k}(t) ; \phi_{k j}$ is the $k-t h$ element of $\phi_{j}$; $G_{j}$ is the complex-valued participation factor associated with the $j-t h$ mode. Eq. (14) shows that each real modal solution is defined as a linear combination of two complex conjugate solutions.

Let us consider now the quantity $\left(\phi_{j}^{T}, s_{j} \boldsymbol{\phi}_{j}^{T}\right) \mathcal{A}\left(\begin{array}{c}\mathbf{u}_{0} \\ \mathbf{v}_{0}\end{array}\right)$ in which $\mathbf{u}_{0}=\mathbf{u}(0)$ and $\mathbf{v}_{0}=\dot{\mathbf{u}}(0)$ are deduced from Eq. (14) and its time-derivative at $t=0$. Then using the $\mathcal{A}$-orthogonality conditions in (A.5), it can be proven that the generic participation factor $G_{j}$ depends on the initial conditions according to the following formula (see e.g. [20]):

$$
G_{j}=\left|G_{j}\right| e^{i \arg G_{j}}=2 \frac{\phi_{j}^{T}\left(s_{j} \mathbf{M} \mathbf{u}_{0}+\mathbf{C} \mathbf{u}_{0}+\mathbf{M} \mathbf{v}_{0}\right)}{\phi_{j}^{T}\left(2 s_{j} \mathbf{M}+\mathbf{C}\right) \phi_{j}}
$$

An alternative form of Eq. (14) is given by the following relationship

$$
u_{k}(t)=\sum_{j=1}^{N} \operatorname{Re}\left(G_{j} \phi_{k j} e^{s_{j} t}\right)=\sum_{j=1}^{N} \operatorname{Re}\left(z^{\left(u_{k j}\right)}(t)\right)
$$

which makes use of the complex time-function $z^{\left(u_{k j}\right)}(t)$ associated with $u_{k j}(t): z^{\left(u_{k j}\right)}(t)=G_{j} \phi_{k j} e^{s_{j} t}$. As a result, it is possible to set

$$
\begin{gathered}
z^{\left(u_{k j}\right)}(t):=A^{\left(u_{k j}\right)}(t) e^{i \alpha^{\left(u_{k j}\right)}(t)} \quad \text { with } \\
A^{\left(u_{k j}\right)}(t)=\left|G_{j}\right|\left|\phi_{k j}\right| e^{-\xi_{j} \omega_{j} t} \\
\alpha^{\left(u_{k j}\right)}(t)=\arg G_{j}+\arg \phi_{k j}+\omega_{j} \sqrt{1-\xi_{j}^{2}} t
\end{gathered}
$$


where the instantaneous amplitude $A^{\left(u_{k j}\right)}(t)$ is a positive and exponentially decreasing time function; the phase $\alpha^{\left(u_{k j}\right)}(t)$ is the sum of three contributions: $\arg G_{j}$ is a constant term related to modal initial conditions; the second term accounts for the complex nature of the mode shapes and it can assume values within the interval $(-\pi, \pi]$; the last term is time-dependent and is related to the modal oscillations of the system. The case of real modes is included in the above expressions: for all $k=1, N$, the imaginary part of $\phi_{k j}$ becomes zero and therefore $\arg \phi_{k j}$ is equal to either 0 or $\pi$ radians, depending on the sign of $R e\left(\phi_{k j}\right)$.

The modal superposition can also be written in terms of velocities

$$
\dot{u}_{k}(t)=\sum_{j=1}^{N} \dot{u}_{k j}(t)=\sum_{i=1}^{N} \frac{1}{2}\left(s_{j} G_{j} \phi_{k j} e^{s_{j} t}+\bar{s}_{j} \bar{G}_{j} \bar{\phi}_{k j} e^{\bar{s}_{j} t}\right)
$$

or in terms of accelerations

$$
\ddot{u}_{k}(t)=\sum_{j=1}^{N} \ddot{u}_{k j}(t)=\sum_{i=1}^{N} \frac{1}{2}\left(s_{j}^{2} \phi_{k j} G_{j} e^{s_{j} t}+\bar{s}_{j}^{2} \bar{\phi}_{k j} \bar{G}_{j} e^{\bar{s}_{j} t}\right)
$$

In these cases, complex representations analogous to (17) for the instantaneous amplitudes and the phases can also be derived:

$$
\begin{gathered}
\dot{u}_{k j}(t)=\operatorname{Re}\left(z^{\left(\dot{u}_{k j}\right)}(t)\right)=A^{\left(\dot{u}_{k j}\right)}(t) \cos \left(\alpha^{\left(\dot{u}_{k j}\right)}(t)\right) \quad \text { with } \\
A^{\left(\dot{u}_{k j}\right)}(t)=\omega_{j} A^{\left(u_{k j}\right)}(t) \\
\alpha^{\left(\dot{u}_{k j}\right)}(t)=\alpha^{\left(u_{k j}\right)}(t)+\arctan \frac{\sqrt{1-\xi_{j}^{2}}}{-\xi_{j}}
\end{gathered}
$$

and

$$
\begin{gathered}
\ddot{u}_{k j}(t)=\operatorname{Re}\left(z^{\left(\ddot{u}_{k j}\right)}(t)\right)=A^{\left(\ddot{u}_{k j}\right)}(t) \cos \left(\alpha^{\left(\ddot{u}_{k j}\right)}(t)\right) \quad \text { with } \\
A^{\left(\ddot{u}_{k j}\right)}(t)=\omega_{j}^{2} A^{\left(u_{k j}\right)}(t) \\
\alpha^{\left(\ddot{u}_{k j}\right)}(t)=\alpha^{\left(u_{k j}\right)}(t)+\arctan \frac{-2 \xi_{j} \sqrt{1-\xi_{j}^{2}}}{-1+2 \xi_{j}^{2}}
\end{gathered}
$$

\section{Signals and continuous wavelet transform: mathematical basis}

In this section, the definitions and main properties of signals and of Continuous wavelet transform are given. The notions useful for identification 
purposes are only introduced; for more mathematical details, see e.g. [21] and $[22]$.

\subsection{Analytic and asymptotic signals}

A real signal $u(t)$ can be defined as a real-valued time function, with $t \in \mathbb{R}$. Square-integrable signals, also called signals of finite energy, are functions belonging to $\mathrm{L}^{2}(\mathbb{R})$. Let $u(t)=A^{(u)}(t) \cos \left[\alpha^{(u)}(t)\right]$ be a given signal of finite energy. The corresponding complex signal is

$$
z^{(u)}(t)=A^{(u)}(t) e^{\alpha^{(u)}(t)}
$$

The pair $\left(A^{(u)}(t), \alpha^{(u)}(t)\right)$ is not unique. However, a reference pair can be defined, which is related to the notion of the analytic signal $Z^{(u)}(t)$ associated with a given signal $u(t)$ :

$$
Z^{(u)}(t)=u(t)+i \mathcal{H}[u](t)=A_{Z}^{(u)}(t) e^{i \alpha_{Z}^{(u)}(t)}
$$

where $\mathcal{H}$ is the Hilbert transform operator. The amplitude-phase pair $\left(A_{Z}^{(u)}(t), \alpha_{Z}^{(u)}(t)\right)$ is called canonical pair and by definition $\dot{\alpha}_{Z}^{(u)}(t)$ is the circular instantaneous frequency of $u(t)$.

The canonical pair is in general different from $\left(A^{(u)}(t), \alpha^{(u)}(t)\right)$, i.e. a signal of the form $A^{(u)}(t) \cos \left[\alpha^{(u)}(t)\right]$ is not in general analytic. However, it can be proven that for signals such that

$$
\left|\frac{\dot{A}^{(u)}(t)}{A^{(u)}(t)}\right| \ll \dot{\alpha}^{(u)}(t)
$$

the following approximations hold

$$
A^{(u)}(t) \simeq A_{Z}^{(u)}(t) \quad \text { and } \quad \alpha^{(u)}(t) \simeq \alpha_{Z}^{(u)}(t)
$$

Signals fulfilling the condition (22) are called asymptotic, as they tend asymptotically to an oscillatory signal without amplitude modulation [23]. In summary, the complex form (20) of asymptotic signals is very close to the associated analytic signal. This kind of signals is simple to analyze by means of wavelet transform [21, pg. 272] and therefore, before using that technique, it is important to verify that signals coming from the free vibration of 
non-proportionally damped linear systems are in fact asymptotic. Indeed, expressions (17) permit to prove that

$$
\left|\frac{\dot{A}^{\left(u_{k j}\right)}(t)}{A^{\left(u_{k j}\right)}(t)}\right| \ll \dot{\alpha}^{\left(u_{k j}\right)}(t)
$$

when $\xi_{j} \ll 1 / \sqrt{2}$. Therefore, free response signals of linear systems with non-proportional viscous damping are asymptotic provided that damping is not too large. Eqs. (18) and (19) lead to analogous proofs for the velocity and acceleration free responses of linear viscously damped systems: they are asymptotic, provided that the damping fulfils the condition $\xi_{j} \ll 1 / \sqrt{2}$.

\subsection{Continuous wavelet transform}

The Continuous Wavelet Transform $(\mathrm{CWT})$ of a real signal $u(t) \in \mathrm{L}^{2}(\mathbb{R})$ is defined as follows:

$$
T_{\psi}[u](b, a)=\frac{1}{a} \int_{-\infty}^{+\infty} u(t) \bar{\psi}\left(\frac{t-b}{a}\right) d t
$$

where $\psi(t) \in \mathrm{L}^{1}(\mathbb{R}) \cap \mathrm{L}^{2}(\mathbb{R})$ is the so-called mother wavelet and $\bar{\psi}$ is its complex conjugate. The parameters $a>0$ and $b \in \mathbb{R}$ introduce scale-dilation and time-translation respectively and thus the wavelet transform makes use of shifted and scaled copies of $\psi(t): \psi_{b, a}(t)=\frac{1}{a} \psi\left(\frac{t-b}{a}\right)$ whose $\mathrm{L}^{1}(\mathbb{R})$ norms $\left(\|\cdot\|_{1}\right)$ are independent from $a$. The linearity of the CWT follows directly from its definition (25). Note that some authors use a different definition of the CWT transform

$$
T_{\psi}[u](b, a)=\frac{1}{\sqrt{a}} \int_{-\infty}^{+\infty} u(t) \bar{\psi}\left(\frac{t-b}{a}\right) d t
$$

which is reported for completeness.

An alternative formulation of the wavelet transform can be obtained by applying Parseval's theorem on (25):

$$
T_{\psi}[u](b, a)=\frac{1}{2 \pi} \int_{-\infty}^{+\infty} \hat{u}(\omega) \hat{\psi}(a \omega) e^{i \omega b} d \omega
$$


where $\hat{\psi}(\omega)=\int_{-\infty}^{+\infty} \psi(t) e^{-i \omega t} d t$ is the Fourier transform of the mother wavelet.

It can also be noted that for $\psi \in C^{2}(\mathbb{R})$ the CWT of velocities and accelerations can be derived from displacements by using the mother wavelets $\dot{\psi}$ and $\ddot{\psi}$, respectively:

$$
\begin{aligned}
& T_{\psi}[\dot{u}](b, a)=-\frac{1}{a} T_{\dot{\psi}}[u](b, a) \\
& T_{\psi}[\ddot{u}](b, a)=-\frac{1}{a} T_{\dot{\psi}}[\dot{u}](b, a)=\frac{1}{a^{2}} T_{\ddot{\psi}}[u](b, a)
\end{aligned}
$$

Eqs. (28) can be derived by integrating by part expression (25). They are useful when displacement data are available but the velocity or acceleration is of interest, since their CWT can be directly evaluated without having to take the derivative of the displacement. Conversely, if acceleration data are available, the following equalities give the CWT of the velocity and displacement

$$
\begin{aligned}
& T_{\psi}[\dot{u}](b, a)=-a T_{\int \psi}[\ddot{u}](b, a) \\
& T_{\psi}[u](b, a)=-a T_{\int \psi}[\dot{u}](b, a)=a^{2} T_{\iint_{\psi}[\ddot{u}](b, a)}
\end{aligned}
$$

where $\int \psi=\int_{-\infty}^{t} \psi(\tau) d \tau$ and $\iint \psi=\int_{-\infty}^{t}\left(\int_{-\infty}^{t^{\prime}} \psi(\tau) d \tau\right) d t^{\prime}$. Using these expressions, no integration has to be performed on the acceleration data.

The $Q$-factor, well-known in conventional frequency analysis of constant $Q$-filters, can be also introduced to characterize the mother wavelet. It is defined as the ratio between the center-frequency and the frequency bandwidth of the mother wavelet (see also the Appendix A.2 and Figure 2)

$$
Q:=\frac{\omega_{\psi}}{2 \Delta \omega_{\psi}}=\frac{\frac{\omega_{\psi}}{a}}{2 \frac{\Delta \omega_{\psi}}{a}}=\frac{\omega_{\psi_{b, a}}}{2 \Delta \omega_{\psi_{b, a}}}
$$

As suggested in [15], it allows a simple comparison between different mother wavelets. Moreover, $Q$ is independent from the time-scale (or time-frequency) point. 


\subsection{Ridge and skeleton of the CWT of asymptotic signals}

In the simplest case of a cosine wave $u_{k j}(t)=A \cos \left(\omega_{0} t\right)$, its wavelet transform is deduced from Eq. (27)

$$
T_{\psi}\left[u_{k j}\right](b, a)=\frac{1}{2}\left(\overline{\hat{\psi}}\left(a \omega_{0}\right) e^{i \omega_{0} b}+\overline{\hat{\psi}}\left(-a \omega_{0}\right) e^{-i \omega_{0} b}\right)
$$

and when the mother wavelet $\psi$ is progressive (see the Appendix A.2), Eq.

(31) becomes

$$
T_{\psi}\left[u_{k j}\right](b, a)=\frac{1}{2} \overline{\hat{\psi}}\left(a \omega_{0}\right) e^{i \omega_{0} b}
$$

The Fourier transform $\hat{\psi}$ of the mother wavelet is assumed to have good localization properties and in particular its absolute value is peaked at the value $\omega=\omega_{\max }$ of the frequency. From Eq. (32), it can be seen that the absolute value $\left|T_{\psi}\left[u_{k j}\right]\right|$ of the wavelet transform is maximum when

$$
a=\frac{\omega_{\max }}{\omega_{0}}
$$

Thus, the CWT has its absolute value localized in the neighborhood of the horizontal line $a=\frac{\omega_{\max }}{\omega_{0}}$ in the time-scale plane and it has the same phase behavior of the signal.

When the CWT is computed according to the definition (26) instead of (25), then (31) becomes

$$
T_{\psi}\left[u_{k j}\right](b, a)=\frac{1}{2} \sqrt{a}\left(\overline{\hat{\psi}}\left(a \omega_{0}\right) e^{i \omega_{0} b}+\overline{\hat{\psi}}\left(-a \omega_{0}\right) e^{-i \omega_{0} b}\right)
$$

In that case, the use of Eq. (33) to identify the value of the angular frequency $\omega_{0}$ leads to a shift (see e.g. [16], [24] and [25]). To correct it, Ta [24] proposed the computation of the maxima of $\left|T_{\psi}\left[u_{k j}\right](b, a)\right|$ after removing the term $\frac{1}{2} \sqrt{a} \overline{\hat{\psi}}\left(-a \omega_{0}\right) e^{-i \omega_{0} b}$ assuming that the mother wavelet is nearly progressive. This leads to solve:

$$
\frac{d\left\{L n\left|\hat{\psi}\left(a \omega_{0}\right)\right|\right\}}{d a}=-\frac{1}{2 a}
$$

which appears to be more suited than Eq. (33). 
Let us consider now a more general form: $u_{k j}(t)=A^{\left(u_{k j}\right)}(t) \cos \left(\alpha^{\left(u_{k j}\right)}(t)\right)$ of an asymptotic signal of finite energy having a single frequency component. Given a progressive mother wavelet $\psi(t)$, it can be proven that a special set of points exists in the time-scale plane $(b, a)$, such that the energy of the CWT of $u_{k j}(t)$ tends to "localize" around it. This set is called the ridge of the $C W T$ of $u_{k j}(t)$. Several definitions of a ridge exist, relying on different criteria of the "localization" of the CWT of $u_{k j}(t)$ around it. The simplest definition postulates that the squared modulus of the CWT (i.e. the energy) is maximized on the ridge [21]:

for every $b$, the condition $\left|T_{\psi}\left[u_{k j}\right](b, a)\right|=\max$

occurs when $\quad a=a_{r}^{\left(u_{k j}\right)}(b)=\frac{\omega_{\max }}{\dot{\alpha}_{Z}^{\left(u_{k j}\right)}(b)} \simeq \frac{\omega_{\max }}{\dot{\alpha}^{\left(u_{k j}\right)}(b)}$

where the ridge is described by its scale value $a_{r}^{\left(u_{k j}\right)}$ as a function of the time variable $b ; \dot{\alpha}^{\left(u_{k j}\right)}(b)$ is the instantaneous circular frequency of the signal. The scale $a$ is related to the instantaneous frequency $\dot{\alpha}^{\left(u_{k j}\right)}(b)$ through the reference frequency $\omega_{\max }$. More sophisticated methods for defining the ridge exist, and the interested reader is referred to [21]. In particular, a phase method was used in [25] to identify the ridge. Moreover, Delprat et al. [26] have shown that the phase methods can be more accurate than amplitude methods.

For asymptotic signals $u_{k j}(t)$ and a progressive mother wavelet $\psi(t)$, the value of the CWT on the ridge can be approximated as follows [23]:

$$
T_{\psi}\left[u_{k j}\right]\left(b, a_{r}^{\left(u_{k j}\right)}(b)\right) \simeq \frac{1}{2} \overline{\hat{\psi}}\left(\omega_{\max }\right) Z^{\left(u_{k j}\right)}(b) \simeq \frac{1}{2} \overline{\hat{\psi}}\left(\omega_{\max }\right) z^{\left(u_{k j}\right)}(b)
$$

Eq. (37) states that the CWT of an asymptotic signal $u_{k j}$ computed on the ridge is proportional to the complex signal $z^{\left(u_{k j}\right)}(b)$.

Now let $u_{k}(t)=\sum_{i=1}^{N} u_{k i}(t)$ be a signal having $N$ distinct frequency components. In this case the CWT has $N$ ridges, one for every frequency component of the signal. On the ridge $j$, the energy of the component $u_{k j}$ is maximized, while the contribution of the other components $u_{k i}, i \neq j$ can be considered negligible if the frequencies are far enough from each other. Then, accounting for Eq. (14), the linearity of the CWT and Eq. (37), one has

$$
\begin{aligned}
T_{\psi}\left[u_{k}\right]\left(b, a_{r}^{\left(u_{k j}\right)}(b)\right) & =T_{\psi}\left[\sum_{i=1}^{N} u_{k i}\right]\left(b, a_{r}^{\left(u_{k j}\right)}(b)\right)=\sum_{i=1}^{N} T_{\psi}\left[u_{k i}\right]\left(b, a_{r}^{\left(u_{k j}\right)}(b)\right) \\
& \simeq T_{\psi}\left[u_{k j}\right]\left(b, a_{r}^{\left(u_{k j}\right)}(b)\right) \simeq \frac{1}{2} \bar{\psi}\left(\omega_{\max }\right) z^{\left(u_{k j}\right)}(b)
\end{aligned}
$$


Eq. (38) shows that it is possible to isolate the frequency component $u_{k j}(b)=\operatorname{Re}\left(z^{\left(u_{k j}\right)}(b)\right)$ from the CWT of a multi-component signal. Then, Eq. (17) can be used to compute the modal properties $\omega_{j}, \xi_{j}$ and $G_{j} \phi_{k j}$ from $u_{k j}(t)$.

\subsection{The modified Cauchy mother wavelet}

The modified Cauchy mother wavelet is defined as follows:

$$
\begin{aligned}
& \psi(t)=\psi_{\beta, n}(t)=\left(\frac{i}{\beta t+i}\right)^{n+1} \\
& \hat{\psi}(\omega)=\hat{\psi}_{\beta, n}(\omega)=2 \pi\left(\frac{\omega}{\beta}\right)^{n} \frac{e^{-\frac{\omega}{\beta}}}{n !} H(\omega)
\end{aligned}
$$

where $H(\cdot)$ is the Heaviside Step function, $n$ is a non-dimensional positive parameter and $\beta$ is a positive parameter whose dimension is the inverse of the dimension of the variable $t$. Note that the usual definition of the (non-modified) Cauchy mother wavelet corresponds to the condition $\beta=1$. It can be readily proven that the modified Cauchy mother wavelet is admissible and progressive. Moreover, the derivatives $\dot{\psi}(t)$ and $\ddot{\psi}(t)$ are related to $\psi(t)$ by the following simple relationships

$$
\begin{aligned}
& \dot{\psi}_{\beta, n}(t)=i \beta(n+1) \psi_{\beta, n+1}(t) \\
& \ddot{\psi}_{\beta, n}(t)=-\beta^{2}(n+1)(n+2) \psi_{\beta, n+2}(t)
\end{aligned}
$$

which allow an easy application of Eqs. (28). Analogously, one has

$$
\int \psi_{\beta, n}(t)=\frac{-i}{\beta n} \psi_{\beta, n-1}(t) \quad \iint \psi_{\beta, n}(t)=\frac{-1}{\beta^{2} n(n-1)} \psi_{\beta, n-2}(t)(41
$$

The $Q$-factor reads

$$
Q=\frac{\sqrt{2 n+1}}{2}
$$

The parameters defining the localization and the uncertainty (see Appendix A.2) are equal to

$$
\begin{array}{ll}
\omega_{\psi}=\frac{\beta}{2}(2 n+1)=2 \beta Q^{2}, & \Delta t_{\psi}=\frac{1}{\beta} \frac{1}{\sqrt{2 n-1}}=\frac{1}{\beta} \frac{1}{\sqrt{4 Q^{2}-2}}, \\
\Delta \omega_{\psi}=\beta \frac{\sqrt{2 n+1}}{2}=\beta Q & \mu_{\psi}=\frac{1}{2} \sqrt{1+\frac{2}{2 n-1}}=\frac{Q}{\sqrt{4 Q^{2}-2}}
\end{array}
$$


and $\omega_{\max }=\beta n=\beta\left(2 Q^{2}-\frac{1}{2}\right)$. For a pure cosine wave of angular frequency $\omega_{0}$, from Eq. (33), the ridge is a horizontal line $a=\frac{\beta n}{\omega_{0}}$. When $\frac{1}{\sqrt{a}}$ is used instead of $\frac{1}{a}$ in the definition of the CWT, Eq. (35) leads to: $a=\frac{\beta\left(n+\frac{1}{2}\right)}{\omega_{0}}$.

In the following sections, only the standard Cauchy mother wavelet $(\beta=1)$ will be used and the CWT definition (25) (or, equivalently, (27)) will be adopted.

\section{An identification technique based on the Wavelet transform}

Provided that the signal coming from free oscillations is asymptotic, it was demonstrated above that it is possible to estimate the modal parameters of interest from the computation of its CWT using Eqs. (17) and (38). In this section, the identification technique based on wavelet analysis which was proposed in [15], is briefly recalled and some improvements are made.

Different practical aspects of the identification process are investigated, with particular efforts devoted to the treatment of edge effects and to the choice of a suitable value of the $Q$-factor characterizing the mother wavelet filtering properties.

\subsection{Edge effects and modal identification}

The so-called edge effects in the CWT computation are related to the fact that in the convolution integral (25), the tails of the mother wavelet $\psi_{b, a}$ are multiplied by the zero values of a causal signal occurring before the initial time $t=0$. For this reason, the relationship (37) between the CWT on the ridge, viz. the skeleton, and the analytic signal is no longer valid and cannot be used for identification purposes. An error appears both in the amplitude and in the phase [16].

For free decay response signals the main edge effect occurs at initial time due to the large initial amplitude value, even though a residual edge effect is also present as $t$ tends to $L$, where $L$ is the finite length of the recorded signal. As shown in Figure 3, the CWT is computed in the time-scale domain

$$
W=\left\{(b, a) \mid b \in[0, L] \quad \text { and } \quad a \in\left[\omega_{\psi} /\left(2 \pi f_{\text {Nyquist }}\right),+\infty\right)\right\}
$$

where the lower limit on $a$ comes from the Nyquist inequality $\omega \leqslant 2 \pi f_{\text {Nyquist }}$ and from the scale-frequency relationship (A.14) discussed in the Appendix A.2 . In our study the CWT computation is performed by solving Eq. (27) 
using the FFT algorithm. Since edge effects are intrinsic in the definition of the CWT, they cannot be completely removed. In order to treat the edge effects, some procedures have been recently proposed. For instance, the shifted [25] Morlet wavelet and the modified Morlet wavelet [17] (also called Gabor wavelet when $\mathrm{L}^{2}$-normalized [16]) allow to modify the frequency resolution around a frequency of interest and also to mitigate edge effects. Moreover, the window reflection method presented by Boltežar and Slavič [27] with reference to the Gabor wavelet can considerably reduce edge effects. In the present contribution, edge effects are not directly reduced, but rather they are adequately controlled, in the sense that when analyzing a ridge, only the portion of it where edge effects are negligible will be considered for further treatment (see also [15] and [16]).

Let $(\bar{b}, \bar{a})$ be a time-scale point. Observe that when $t_{\psi}=0$, the mother wavelet modulus $\left|\psi_{\bar{b}, \bar{a}}(t)\right|$ is maximum at the mean value $\bar{b}$ and vanishes far away from it. Let $c_{t}$ be a positive coefficient such that the CWT computed at $(\bar{b}, \bar{a})$ by means of (25), or equivalently (27), depends mainly on the signal values $u(t)$ occurring for the interval

$$
t \in \mathbb{I}(\bar{b}, \bar{a})=\left[\bar{b}-c_{t} \Delta t_{\psi} \bar{a}, \bar{b}+c_{t} \Delta t_{\psi} \bar{a}\right]
$$

Since the values of $u(t)$ for $t \notin \mathbb{I}(\bar{b}, \bar{a})$ are multiplied by the tails of the mother wavelet in the convolution product (25), they do not affect the CWT computation substantially. Therefore, if the zero $u$-values occurring for $t<0$ overlap with the tails of $\psi_{\bar{b}, \bar{a}}$, the edge effects at $(\bar{b}, \bar{a})$ can be considered small. The greater the value $c_{t}$ is, the smaller the mother wavelet tails are and the smaller the residual edge effects at $(\bar{b}, \bar{a})$ are.

The choice of a suitable $c_{t}$ value, i.e. a value such that the residual edge effects at $(\bar{b}, \bar{a})$ do not affect the modal identification substantially, has already been discussed in [16], with reference to the Gabor mother wavelet. A more general analysis is proposed here, aiming to estimate the $c_{t}$ value without making reference to a specific mother wavelet. Let us now apply the Gauss-Winkler inequality [28, pp. 238-240] to the probability distribution function $\tilde{\psi}_{\bar{b}, \bar{a}}(t)=\frac{\left|\psi_{\bar{b}, \bar{a}}(t)\right|^{2}}{\left\|\psi_{\bar{b}, \bar{a}}\right\|_{2}^{2}}$ associated with a generic mother wavelet $\psi_{\bar{b}, \bar{a}}(t)$. This probability density function, defined in the Appendix A.2, has a mean value equal to $\bar{b}$ and standard deviation equal to $\bar{a} \Delta t_{\psi}$. The sum $\varepsilon_{t}$ of the 
two tail areas of $\tilde{\psi}_{\bar{b}, \bar{a}}(t)$ is upper-bounded as follows

$$
\begin{aligned}
\varepsilon_{t}= & \operatorname{Prob}\left[|t-\bar{b}| \geq c_{t}\left(\bar{a} \Delta t_{\psi}\right)\right] \\
& \leq \begin{cases}1-\frac{c_{t}}{\sqrt{3}} & c_{t} \leq \frac{2}{\sqrt{3}} \simeq 1.155 \text { or } \varepsilon_{t} \geq 1 / 3 \\
\frac{4}{9} \frac{1}{c_{t}^{2}} & c_{t} \geq \frac{2}{\sqrt{3}} \simeq 1.155 \text { or } \varepsilon_{t} \leq 1 / 3\end{cases}
\end{aligned}
$$

When $\psi$ is even, $\varepsilon_{t}$ in Eq. (44) can be written as $\varepsilon_{t}=2 \operatorname{Prob}\left[t-\bar{b} \geq c_{t}\left(\bar{a} \Delta t_{\psi}\right)\right]$. By fixing a priori the tail area $\varepsilon_{t}$, relationships (44) allow the computation of a value of $c_{t}$, which is independent from the form of $\psi_{\bar{b}, \bar{a}}(t)$. A small tail area $\varepsilon_{t}$ corresponds to small residual edge effects at $(\bar{b}, \bar{a})$ and leads to a large $c_{t}$ value. For instance, if $\varepsilon_{t}=0.018$, then $c_{t} \simeq 5$.

If the explicit expression of $\psi_{\bar{b}, \bar{a}}(t)$ is known, then the $c_{t}$ value corresponding to the tail area $\varepsilon_{t}$ can be evaluated exactly. This is the case for the Cauchy mother wavelet, where the $c_{t}-\varepsilon_{t}$ relationship is depicted in Figure 4a. The value $c_{t}=5$ corresponds to values of $\varepsilon_{t}$ much smaller than 0.018 , as illustrated in Figure 4b.

Consider now the set $W$ of all time-scale points $(b, a)$. The edge effects are small only in the reduced region defined as

$$
\begin{aligned}
& W_{\text {red }}=\{(b, a) \text { such that } \mathbb{I}(b, a) \subseteq W\} \\
& =\left\{(b, a) \text { such that } b-c_{t} \Delta t_{\psi} a \geq 0 \text { and } b+c_{t} \Delta t_{\psi} a \leq L\right\}
\end{aligned}
$$

Recalling Eq. (A.14), one can assume $t=b$ and $\omega=\omega_{\psi} / a$ and the sets $W$ and $W_{\text {red }}$ can be defined in the time-frequency plane by :

$$
\tilde{W}=\left\{(t, \omega) \mid t \in[0, L] \text { and } \omega \in\left[0,2 \pi f_{\text {Nyquist }}\right]\right\}
$$

and

$$
\begin{aligned}
& \tilde{W}_{\text {red }}=\left\{(t, \omega) \in \tilde{W} \quad \text { such that } \mathbb{I}\left(t, \frac{\omega_{\psi}}{\omega}\right) \subseteq W\right\} \\
& =\left\{(t, \omega) \in \tilde{W} \quad \text { such that } t \geq c_{t} \Delta t_{\psi} \frac{\omega_{\psi}}{\omega} \text { and } t \leq L-c_{t} \Delta t_{\psi} \frac{\omega_{\psi}}{\omega}\right\}
\end{aligned}
$$

Expression (46) indicates that the effective time-frequency window $\tilde{W}_{\text {red }}$ is delimited by two hyperbolae (see Figure $3 \mathrm{~b}$ ) and an equivalent expression can be derived from relationships (A.13) and (30)

$$
\tilde{W}_{\text {red }}=\left\{(t, \omega) \in \tilde{W} \quad \text { such that } t \geq \frac{c_{t} Q 2 \mu_{\psi}}{\omega} \quad \text { and } \quad t \leq L-\frac{c_{t} Q 2 \mu_{\psi}}{\omega}\right\}
$$




\subsection{Effective stopping time for modal identification}

Free oscillations in a SDoF linear system are characterized by the product of the modal damping ratio and the angular eigenfrequency, which is a measure of how fast the signal amplitude decreases. For MDoF linear systems, the coexisting modes are associated with distinct modal products, hence they die out at different rates. Thus, after a certain time period depending on the modal product and on the initial modal amplitude, some vibration modes can get damped out completely, which means that the residual vibration energy in them becomes so small that their characteristic ridges on the time-frequency plot become very difficult to extract.

To remedy this difficulty, a stopping time $t_{\bar{U}_{k j}}$ is introduced. It is defined as the time when the decreasing signal amplitude reaches a given threshold $\bar{U}_{k j}$, for each recorded signal $u_{k}\left(k \in\left[1, N_{s}\right]\right)$ and each ridge corresponding to a modal frequency $\omega_{j}\left(j \in\left[1, N_{f}\right]\right.$, where $N_{f}$ is the number of identified modal frequencies). In practice, the threshold value is chosen to be the same for all recorded signals $\left(k=1, N_{s}\right)$ at a given modal frequency $j$. For real experimental data, the choice of the value of $\bar{U}_{j}$ depends on the signal to noise ratio, which is in turn related to the sensors, the cables and the overall test set-up. For numerically simulated signals, rounding-up errors may occur and in this case, a suitable threshold value also needs to be adopted as shown in the numerical examples of Section 5.

\subsection{Discussion on the choice of an optimal $Q$-value for the mother wavelet}

Three aspects of the identification problem mainly need to be accounted for in the choice of a suitable $Q$-value for the CWT analysis of a given signal: (i) the presence of modes close in frequency, (ii) the need to minimize the influence of edge effects and (iii) the characteristics of the analyzed signal. A specific bound on the $Q$ value is computed for each of the above three cases, from which an optimal value of $Q$ is later deduced.

(i) Let us begin with an analysis of the case in which close modes occur, i.e. when there are two modal circular frequencies $\omega_{j}$ and $\omega_{j}+d \omega$, with $d \omega$ small. To get correct identification results for both modes, the mutual influence of two close frequencies must be negligible in the CWT computation. This condition is fulfilled when $\hat{\psi}_{b, a}(\omega)$ is such that it has a maximum value at one of the two frequencies and a much lower value for the other frequency. Hence, it is sufficient to impose the following 
condition on the standard deviation of $\hat{\psi}_{b, a}(\omega)$ :

$$
d \omega \geq c_{f} \Delta \omega_{\psi_{b, a}}=c_{f} \frac{\omega_{j}}{\omega_{\psi}} \Delta \omega_{\psi}=c_{f} \frac{\omega_{j}}{2 Q}
$$

leading to the first inequality for $Q$

$$
Q \geq Q_{\min }=c_{f} \frac{\omega_{j}}{2 d \omega}
$$

The coefficient $c_{f}$ associated to $\Delta \omega_{\psi_{b, a}}=\Delta \omega_{\psi} / a$ plays the same role as $c_{t}$ for the time standard deviation $\Delta t_{\psi_{b, a}}=a \Delta t_{\psi}$.

(ii) It was previously found that there exists a time interval smaller than the whole recording time interval $[0, L]$, where the edge effects can be negligible. For a given circular frequency $\omega_{j}$, this interval can be denoted $\left[t_{1, j}, L-t_{1, j}\right]$, where $t_{1, j}$ is derived from the bounds given in (47), for $\omega=\omega_{j}$ :

$$
t_{1, j}=\frac{c_{t} Q 2 \mu_{\psi}}{\omega_{j}}
$$

This interval exists if $0 \leq t_{1, j}<\frac{L}{2}$, leading to the following inequality

$$
Q \leq Q_{\max }=\frac{\omega_{j} L}{4 c_{t} \mu_{\psi}},
$$

This relationship shows that the upper bound $Q_{\max }$ on the $Q$-value depends both on the length $L$ of the recorded interval, on the edge effects through $c_{t}$ and on the analyzed frequency $\omega_{j}$. In order to have a non-empty set of effective $Q$-values, $Q_{\min }<Q_{\max }$ must hold. When this is not the case, then either $c_{f}, c_{t}$ or $L$ have to be changed.

Moreover $t_{1, j}$ must be lower than the stopping time $t_{\bar{U}_{k j}}$ introduced in 4.2 for at least one of the signals $u_{k j}\left(1 \leq k \leq N_{s}\right)$ :

$$
t_{1, j}<t_{\bar{U}_{k j}}
$$

Otherwise, $t_{1, j}$ must be reduced, by either reducing $c_{t}$ or choosing a smaller $Q$. The number of signals satisfying the condition (52) is indicated as $\tilde{N}_{s}$, with $1 \leq \tilde{N}_{s} \leq N_{s}$. Hence, the effective time intervals $\mathbb{I}_{k j}=\left\{t_{1, j}, t_{\bar{U}_{k j}}\right\}$, $k=1, \tilde{N}_{s}$ are proposed as the most appropriate time intervals for performing the frequency and damping identification. On the other hand, the mode shape identification is carried out by considering all the $N_{s}$ signals on the same time interval $\mathbb{I}_{j}=\left[t_{1, j}, \min _{k=1, \tilde{N}_{s}}\left(t_{\bar{U}_{k j}}\right)\right]=\left[t_{1, j}, t_{\bar{U}_{j}}\right]$.

(iii) The set of effective values of $Q$ could be further narrowed down by taking into account the way the amplitude $A(t)$ of the analyzed signal $u(t)$ decreases. Let us consider the free response of a linear viscous SDoF system with natural frequency $\omega$ and damping ratio $\xi$; its amplitude is 
exponentially decreasing and causal: $A(t)=A_{0} e^{-\xi \omega t} H(t)$ where $H(t)$ is the Heaviside function. Let us define in a similar way the even amplitude $\tilde{A}(t)=A_{0} e^{-\xi \omega|t|}$. Its standard deviation $\Delta t_{\tilde{A}}$ whose definition is given in relation (A.12) for $\psi(t)$, can be derived

$$
\Delta t_{\tilde{A}}=\frac{1}{\sqrt{2} \xi \omega}
$$

It has been proven in [29] that when the amplitude $A(t)$ is a Gaussian function and the mother wavelet $\psi(t)$ is related to a Gaussian window, the mother wavelet ensuring entropy minimization fulfils the relationship $\Delta t_{\psi_{b, a}}=\Delta t_{A}$. Based on numerical simulations, it can be shown that this condition becomes an inequality when $A(t)=\tilde{A}(t)$ and $\psi(t)$ is the Cauchy mother wavelet

$$
\Delta t_{\psi_{b, a}} \leq \Delta t_{\tilde{A}}
$$

Eq. (54) leads to the third inequality for $Q$

$$
Q \leq Q_{\xi}
$$

For the Cauchy mother wavelet (see Eqs. (A.15) and (42)), the expressions (53) and (55) lead to the explicit computation of $Q_{\xi}$ which depends only on $\xi$ :

$$
Q_{\xi}=\frac{1}{2 \xi} \sqrt{1+\sqrt{1-4 \xi^{2}}}
$$

provided that $\xi \leq \frac{1}{2}$.

For $\xi \ll \frac{1}{2}$, the approximate expression $Q_{\xi}=\frac{1}{\sqrt{2} \xi}$ can be used, which implies that when the damping ratio tends to zero, the value of $Q_{\xi}$ tends to infinity. The choice of $Q$ infinite corresponds to an infinite frequency-resolution and a zero time-resolution, which is the situation for the standard Fourier transform.

For experimental data, the value of $\xi$ has to be initially estimated from the Fourier transform of the recorded signal, for instance by using the half-power rule. Once $Q_{\xi}$ is computed using Eq. (56), the subsequent CWT analysis will provide a better estimation of $\xi$. Numerical simulations indicate that when

$$
Q=\frac{Q_{\min }+Q_{\max }}{2}<Q_{\xi}
$$

a precise modal identification can be performed [15], accounting for the fact that smaller $Q$ values lead to greater time resolution and smaller frequency resolution. For high damping ratios, this upper limit may become quite small and in particular smaller than $Q_{\max }$. This situation is illustrated through the numerical examples presented in the last section. 
In summary, there are three reference values of $Q$, namely $Q_{\min }, Q_{\max }$ and $Q_{\xi}$. The inequality $Q_{\min }<Q_{\max }$ must always hold. In addition, two cases have to be distinguished:

(a) $Q_{\min } \leq Q_{\xi}$;

(b) $Q_{\min }>Q_{\xi}$

In the first case, one can set $Q=\min \left(Q_{\xi}, 1 / 2\left(Q_{\min }+Q_{\max }\right)\right)$. In the second case, the correct choice is $Q=Q_{\min }$.

\subsection{Least squares estimate of the modal properties}

The modal identification can be performed in the following three steps:

(1) By definition (see Eq. (36)), every recorded signal produces one ridge centered around each eigenfrequency of the system. On a time-frequency plot these ridges illustrate the time-evolution of its corresponding instantaneous frequency. In principle, for a linear system all these instantaneous frequencies should be constant in time and equal to the modal pseudo-frequency $2 \pi \tilde{f}_{j}=\tilde{\omega}_{j}=\omega_{j} \sqrt{1-\xi_{j}^{2}}$. In practice, they are often distinct and/or varying. Therefore, the unique identified value is defined as the average (in time and on the $\tilde{N}_{s}$ signals) of all the instantaneous frequencies. This corresponds to adopting a least square minimization procedure identification:

$$
\begin{array}{r}
\tilde{\omega}_{j}=\arg \left[\min F_{1}\left(\tilde{\omega}_{j}\right)\right] \quad \text { with } \\
\begin{aligned}
F_{1}\left(\tilde{\omega}_{j}\right) & =\sum_{k=1}^{\tilde{N}_{s}} \int\left(\frac{\omega_{\max }}{a_{\mathbb{I}_{k j}}^{\left(u_{k j}\right)}(b)}-\tilde{\omega}_{j}\right)^{2} d b \\
& =\sum_{k=1}^{\tilde{N}_{s}} \int_{\mathbb{I}_{k j}}\left(\dot{\alpha}^{\left(u_{k j}\right)}(b)-\tilde{\omega}_{j}\right)^{2} d b
\end{aligned}
\end{array}
$$

(2) The modal damping ratio $\xi_{j}$ can be identified by using the fact that the analytic signals $Z_{u_{k j}}(t)$ extracted from the ridges have an exponential decay. By adopting a least squares procedure, one obtains

$$
\begin{aligned}
& \left(\xi_{j} \omega_{j},\left|Q_{j}\right|\left|\phi_{k j}\right|\right)=\arg \left[\min F_{2}\left(\xi_{j} \omega_{j},\left|Q_{j}\right|\left|\phi_{k j}\right|\right)\right] \\
& F_{2}\left(\xi_{j} \omega_{j},\left|Q_{j}\right|\left|\phi_{k j}\right|\right) \\
& \quad=\sum_{k=1}^{N_{\mathbb{I}_{k j}}} \int_{\mathbb{I}_{k j}}\left(\ln \left|Z_{u_{k j}}(b)\right|-\left(\ln \left(\left|Q_{j}\right|\left|\phi_{k j}\right|-\xi_{j} \omega_{j} b\right)\right)\right)^{2} d b
\end{aligned}
$$


The parameters $\xi_{j} \omega_{j}$ and $\left|Q_{j}\right|\left|\phi_{k j}\right|$ can be identified. Then, by using the $\tilde{\omega}_{j}$ value identified at step (1), it is possible to compute $\xi_{j}$ and $\omega_{j}$.

(3) Let $Z_{u_{l j}}(b)$ and $Z_{u_{k j}}(b)$ be the analytic signals identified by the CWT for the frequency $\tilde{f}_{j}$, at reference point $l$ and at the generic point $k$ of the structure, respectively. Moreover, let introduce the instantaneous ratio between $Z_{u_{k j}}(b)$ and $Z_{u_{l j}}(b): \frac{Z_{u_{k j}}(b)}{Z_{u_{l j}}(b)}$. Then, the complex vector $\mathbf{Z}_{u_{j}}(b)=\left[\frac{Z_{u_{1 j}}(b)}{Z_{u_{l j}}(b)}, \ldots, \frac{Z_{u_{N j}}(b)}{Z_{u_{l j}}(b)}\right]^{T}$ defined by putting together all these ratios can be interpreted as an estimate of the time-evolution of the complex mode shape $\phi_{j}$, normalized with respect to the chosen reference point. Therefore, the mode shape is identified by the following minimization procedure

$$
\begin{aligned}
& \boldsymbol{\phi}_{j}=\arg \left[\min F_{3}\left(\phi_{j}\right)\right] \quad \text { with } \\
& F_{3}\left(\boldsymbol{\phi}_{j}\right)=\int_{\mathbb{I}_{j}}\left\|\mathbf{Z}_{u_{j}}(b)-\phi_{j}\right\|^{2} d b
\end{aligned}
$$

The different phases of the CWT analysis and of the modal identification are summarized below:

(1) Pre-treatment of data

(i) Define the type of signals (displacements, velocities or accelerations).

(ii) Compute the Fourier transforms $\hat{u}_{k}(f)$ for $k=1, \ldots, N_{s}$ and estimate the peak frequencies $\tilde{f}_{j}=\frac{\tilde{\omega}_{j}}{2 \pi}$ and the modal damping ratios $\xi_{j}$.

(2) Choice of the parameters for the CWT analysis, at the frequency $\tilde{f}_{j}$.

(i) Choose $c_{t}, c_{f}$ and set the threshold value $\bar{U}_{j}$.

(ii) Define the distance $d \omega$ between the analyzed circular frequency $\tilde{\omega}_{j}=2 \pi \tilde{f}_{j}$ and the one closest.

(iii) Compute $Q_{\min }, Q_{\max }$ and $Q_{\xi}$. Choose $Q$. Deduce $n$ for the Cauchy wavelet.

(3) CWT analysis: computation of ridges and analytic signals of $u_{k}(t)$ for $k=1, \ldots, N_{s}$, at the frequency $\tilde{f}_{j}$.

(i) Define the frequency interval of the CWT analysis around $\tilde{f}_{j}$ and choose the number of frequency lines within the interval.

(ii) Compute the CWT in terms of displacements, by using either (27) or $(29)_{1,2}$ depending on whether the measured signals are displacements, velocities or accelerations.

(iii) Extract the ridges $a_{r}^{\left(u_{k j}\right)}(b)$, with their effective time intervals $\mathbb{I}_{k j}$ and the analytic signals $Z_{u_{k j}}(b)$, by using Eqs. (36) and (37), respectively.

(4) Modal parameter identification

(i) Identification of the modal frequency (Eq. (58)). 
(ii) Identification of the damping ratio (Eq. (59)).

(iii) Identification of the complex mode shape (Eq. (60)).

(5) Choose another frequency $\tilde{f}_{j}$ and return to step 2.

\section{Validation by numerical simulations}

In this section, two numerical examples are presented in order to illustrate the efficiency of the proposed identification technique. These examples have been specifically chosen to have high modal damping ratios and high non-proportionality indices for the complex modes. In all cases, after-shock free vibration responses of a four DoFs system are analyzed, where the shock is introduced in the form of an initial velocity. The numerical simulation is performed by using the ODE45 Matlab solver, based on an explicit Runge-Kutta time-stepping algorithm. The chosen time step is $\Delta t=0.1163$ $\mathrm{s}$ and the signal length is $L=(2048-1) \Delta t=238.0233 \mathrm{~s}$. The Nyquist frequency is thus: $f_{N y}=\frac{1}{2 \Delta t}=4.3 \mathrm{~Hz}$. The CWT analysis is performed on the whole recorded time interval and on local frequency bands having an amplitude of $0.2 \mathrm{~Hz}$ (except for the last mode in the second example) and divided in 500 frequency lines. The $c_{t}$ and $c_{f}$ values are chosen between 4.0 and 5.8. As a result, as proven in the analysis of Section 4.1, the residual edge effects are very small.

In the first example, the maximum modal damping is around $10 \%$ (first mode) and the maximum non-proportionality index is around 0.27 for the fourth mode, whose frequency is close to that of the third one. Two cases are examined: signals with and without added white noise having a standard deviation of $\sigma_{n}=10^{-3}$. All modes are identified with a very good accuracy in both cases. In the second example, the damping of the first mode is around $35 \%$. In addition, the non-proportionality index for the last two modes, which have frequencies close each other, is greater than 0.3. Under these conditions, the identification remains very good in the noise-free case. However when noise having the same characteristics as the one in the previous example is added, the identification of the last mode becomes impossible since the signal amplitude is less than that of the noise. 


\subsection{Example 1}

The system in this first example has 4 DoFs (Fig. 5) and is characterized by the following mass and stiffness matrices:

$$
\mathbf{M}=\left[\begin{array}{llll}
1 & 0 & 0 & 0 \\
0 & 2 & 0 & 0 \\
0 & 0 & 2 & 0 \\
0 & 0 & 0 & 1
\end{array}\right] \quad \mathbf{K}=\left[\begin{array}{cccc}
5 & -3 & 0 & 0 \\
-3 & 7 & -4 & 0 \\
0 & -4 & 7 & -3 \\
0 & 0 & -3 & 5
\end{array}\right]
$$

The modal properties of the associated undamped system are shown in Table 1. The damping matrix is

$$
\mathbf{C}=\left[\begin{array}{cccc}
C_{1} & 0 & 0 & 0 \\
0 & C_{2} & 0 & 0 \\
0 & 0 & 0.2 & 0 \\
0 & 0 & 0 & 0.1
\end{array}\right]
$$

The application of the proportionality condition (11) gives $C_{1}=0.1$ and $C_{2}=0.2$, leading to a damping matrix proportional to the mass matrix (Rayleigh condition). Therefore the modes are real and the non-proportionality index $I_{n p}^{(j)}$ is zero for all modes.

When the damping parameters are chosen as follows

$$
C_{1}=0.5, C_{2}=0.2
$$

condition (11) is not fulfilled and therefore the mode shapes are complex. The modal properties of this system are collected in Table 2. Observe that the second column consists of the pseudo-frequencies $\tilde{f}_{j}=f_{j} \sqrt{1-\xi_{j}^{2}}$ instead of the natural frequencies. This choice is motivated by the fact that the ridge extraction of the CWT analysis leads directly to the identification of the pseudo-frequencies.

The modal analysis of this system is performed by using numerical free vibration responses, where shocks are introduced under the form of an initial velocity $v_{0}=1 \mathrm{~m} / \mathrm{s}$ applied only to the fourth mass. For all modes, the threshold value has been set to $\bar{U}_{j}=10^{-5} \mathrm{~m}$. For the first two modes, the $Q$-factor has been chosen to be equal to $Q_{\xi}$, leading to a good balance 
between time and frequency resolution (see Table 3). The third and fourth modes are close to each other in frequency and the best choice is $Q=Q_{\text {min }}$, since the separation of the two frequency peaks is ensured by a small value of $\Delta \omega_{\psi_{b, a}}$ (see Table 4). If the lower value $Q=Q_{\xi}$ had been chosen, the frequency resolution would have been too low. The results presented in Table 5 show a very good correspondence between the identified and the exact modal properties. Figure 6 illustrates in greater detail the extracted ridges and skeletons as well as the identified complex mode shape for the first mode. The effective time-interval of the analysis $\mathbb{I}_{j}$ is also indicated. Similar plots might be reported for the other modes, but they are omitted for brevity. Figure 7 shows for the second mode the error in each identified parameter as a function of $Q$, for fixed values of $c_{t}, c_{f}$ and $\bar{U}_{j}=\bar{U}_{2}$. It can be seen that there is a relatively large interval of $Q$-values, between $Q_{\min }=7$ and $Q \simeq 17$, where the three errors remain less than $0.1 \%$. One can also note that the size of the effective time interval decreases as $Q$ increases. The y-plot ranges of this Figure has been chosen to allow an easy comparison to Figure 8, which gives the same plots of the identification errors for the third mode. In this case, the importance of the choice of $Q$ can be observed with respect to the presence of close frequencies. When $Q<Q_{\min }=26.9$ the errors increase rapidly and become very large. Recall that $Q_{\min }$ is related to the frequency distance between the third and fourth close modes. The non-proportionality index $\tilde{I}_{n p}^{(j)}$ is also correctly estimated for each mode $j$ (see Tables 2 and 5 for the exact and the identified values respectively).

When white noise is added (see Figure 9 for the first mode), the threshold value $\bar{U}_{j}$ also needs to be changed, in such a way that $\bar{U}_{j}>\sigma_{n}$. As a result, $t_{\bar{U}_{j}}$ is reduced along with $c_{t}$ and $c_{f}$ as shown in Table 6 . The identification leads to very small errors (Table 7 ).

\subsection{Example 2}

The second example is chosen in order to test the performance of the CWT modal analysis on a system having very high damping and close modes with very high non-proportionality index. The mass and stiffness matrices are the same as in (61). The damping matrix is given by (62) with $C_{11}=0.1$ and $C_{22}=2.2$. All the relevant data are reported in Table 8 . Note that the non-proportionality indices of all modes are high, especially for the last two modes, characterized by close frequencies.

The shock $\left(v_{0}=1 \mathrm{~m} / \mathrm{s}\right)$ that causes the free vibration responses is applied at node 2 . For the first three modes, the threshold value has been set to $\bar{U}_{j}=10^{-5} \mathrm{~m}$, with $j=1,2,3$ and for the last one $\bar{U}_{4}=10^{-6} \mathrm{~m}$ (see Table 9 ). Like in the Example 1, the third and fourth modes are close to each other in 
frequency and the best choice is $Q=Q_{\min }$, since the separation of the two frequency peaks is ensured by a small value of $\Delta \omega_{\psi_{b, a}}$ (see Table 10). The results of the identification procedure are listed in Table 11. For the first mode of circular frequency $\tilde{\omega}_{1}=2 \pi \tilde{f}_{1}$, the value of $Q$ is set to $Q_{\min }=\frac{c_{f}}{2}$ through Eq. (49) where $d \omega=\tilde{\omega}_{1}$. The damping is very high and the condition of asymptoticity (24) is no longer completely fulfilled. This implies that the ridge is no longer straight (Figure 10). However, a good identification of the frequency as well as of the damping ratio and the mode shape is still possible, as shown in Table 11. For the second mode, the identification is obtained with $Q$ very close to $Q_{\xi}$, which is given in Eq. (56), and in this case is slightly greater than $Q_{\min }$ (see Table 9). The results remain very accurate with a relative error of less than $0.12 \%$ (see Figure 12). The last two modes (the fourth one is described by Figure 11) are close in frequency and both have a significant modal damping ratio. The $Q$-value is set to $Q_{\min }$ in both cases. The errors in the identification of the damping and of the complex mode shapes remain acceptable, yet they are larger than that obtained in the Example 1. This discrepancy is due to the coupling of the modes combined with the fact that the two modes are not well excited by the shock applied to only one mass. When noise is added, the identification is still satisfactory for the first three modes, while the fourth one cannot be identified (see Tables 12, 13 and Figure 14).

\section{Application to a plexiglass plate}

The modal identification technique is applied to acceleration signals recorded on a rectangular plexiglass plate of width $39.7 \mathrm{~cm}$ (x-axis), length $49 \mathrm{~cm}$ (y-axis) and thickness $0.41 \mathrm{~cm}$. A finite element model of the plate has been implemented, with a mass density of $1218.2 \mathrm{~kg} / \mathrm{m}^{3}$, a Young modulus of $5150 \mathrm{MPa}$ and Poisson ratio equal to 0.41 . The modal frequencies of this undamped model are listed in Table 15. The mode type is indicated in the second column by the indices $(m, n)$, where $m(n)$ is the number of modal lines orthogonal to the $\mathrm{x}(\mathrm{y})$-axis . The test set-up has been conceived in such a way to ensure that the plate vibration is free in the direction orthogonal to its mean plane. The shocks are introduced by tapping the plate with a small steel bar at the points 1 and 2 indicated in Figure 15. The sampling time step between two data points is $\Delta t=0.001953 \mathrm{~s}$ and the signal length is $L=(1200-1) \Delta t=2.3418 \mathrm{~s}$. The Nyquist frequency is thus $f_{N y}=\frac{1}{2 \Delta t}=256 \mathrm{~Hz}$. Four accelerometers were available for the experiments. For each shock point, six sets of measurements were taken, each time changing the position of three of the sensors while maintaining the first one always at the same place, in order to have a reference signal. $N_{s}=24$ signals were analyzed, leading to the identification of ten modes (Tables 14 and 15). 
Figures 16, 17, 18, 20 and 21 illustrate the identification results for the modes 1, 2, 5, 8 and 10, respectively. Observe that the second mode shape has a significant imaginary part. A particular analysis has been required for the identification of the mode 7 , having almost the same frequency as mode 8. As a matter of fact, when the shock inducing the free vibration is imposed at the point 1 , the mode 8 is excited with a far large amplitude than the mode 7 . Conversely, when the shock is imposed at the point 2, lying approximately on a nodal line of the mode 8 , only the mode 7 is excited at that frequency, and its identification becomes easy (Figure 19). In addition, the high frequency modes 9 and 10 are well-excited when imposing the shock at the point 2. Hence, the values of frequency and damping of these modes have been identified also on this second data set (see the last three columns of Table 15). Note that the non-proportionality indices $\tilde{I}_{n p}^{(j)}$ have been computed for all modes of the plate.

\section{Conclusions}

An effective modal identification technique based on the use of the CWT was proposed. Free vibration responses of non-proportionally damped systems were analyzed and modal frequencies, damping ratios and complex mode shapes were accurately identified. The edge effects were adequately accounted for and several numerical simulations validated the technique for a large range of modal damping ratios and large non-proportionality indices of the modes. The identification accuracy at significant damping, where the asymptoticity of the free decay responses does not necessary hold, was also discussed. In addition the practical aspects concerning the selection of an optimal mother wavelet were investigated and an expression for the optimal $Q$-factor of the mother wavelet as function of the damping ratio was proposed and validated by numerical simulations and applied to the modal identification of a plexiglass plate.

\section{Acknowledgements}

The authors would like to thank Georges Nikiforov for his assistance in improving the English of this manuscript. 


\section{A Appendix}

\section{A.1 Non-proportional damping}

\section{A.1.1 Modal frequencies and damping ratios}

Eq. (7) can be normalized in such a way that the mass matrix $\mathbf{M}$ in front of $s^{2}$ is transformed into the identity matrix $\mathbf{I}_{N}$ of $\mathbb{R}^{N}$. In order to do that, observe that as $\mathbf{M}$ is a real symmetric and positive definite matrix, a real transformation $\mathbf{T}$ always exists so that

$$
\mathbf{T}^{T} \mathbf{M T}=\mathbf{I}_{N}
$$

with

$$
\mathbf{T}=\boldsymbol{\Theta}_{m} \mathbf{W}, \quad \boldsymbol{\Theta}_{m}^{T} \boldsymbol{\Theta}_{m}=\mathbf{I}_{N}, \quad \mathbf{W}=\operatorname{diag}\left(1 / \sqrt{\lambda_{m, j}}\right)
$$

where $\mathbf{T} \mathbf{T}^{T}=\mathbf{M}^{-1}, \lambda_{m, j}$ are the positive and real eigenvalues of $\mathbf{M}$ and $\boldsymbol{\Theta}_{m}=\left(\boldsymbol{\theta}_{m, 1}, \ldots, \boldsymbol{\theta}_{m, N}\right)$ is the matrix whose columns are the corresponding orthonormal eigenvectors. Then, setting

$$
\phi=\mathbf{T v}
$$

and pre-multiplying (7) by the transpose of $\mathbf{T}$, one obtains the normalized expression

$$
\left(s^{2} \mathbf{I}_{N}+s \mathbf{A}+\mathbf{B}\right) \mathbf{v}=\mathbf{0}
$$

with

$$
\mathbf{T}^{T} \mathbf{C T}=\mathbf{A}, \quad \mathbf{T}^{T} \mathbf{K T}=\mathbf{B}
$$

Note that both matrices $\mathbf{A}$ and $\mathbf{B}$ are real, symmetric and non-negative.

The characteristic values associated with (5) (as well as (7) and (A.1)) occur in complex conjugated pairs. In order to show that, consider the generic characteristic pair $\left(s_{j}, \mathbf{v}_{j}\right)$ in Eq. (A.1) and pre-multiply the same equation by $\overline{\mathbf{v}}_{j}^{T}$, where the superposed bar indicates complex conjugation:

$$
s_{j}^{2} \overline{\mathbf{v}}_{j}^{T} \mathbf{v}_{j}+s_{i} \overline{\mathbf{v}}_{j}^{T} \mathbf{A} \mathbf{v}_{j}+\overline{\mathbf{v}}_{j}^{T} \mathbf{B} \mathbf{v}_{j}=\mathbf{0}
$$


Each eigenvector $\mathbf{v}_{j}$ is then normalized so that its standard Hermitian norm on $\mathbb{C}^{N}:\left\|\mathbf{v}_{j}\right\|^{2}=\overline{\mathbf{v}}_{j}^{T} \mathbf{v}_{j}$ is equal to one. Moreover, since $\mathbf{A}$ and $\mathbf{B}$ are real, symmetric and non-negative matrices, let us introduce $\lambda_{a, j}$ and $\lambda_{b, j}$, the real and non-negative eigenvalues of $\mathbf{A}$ and $\mathbf{B}$ respectively. $\boldsymbol{\theta}_{a, j}$ and $\boldsymbol{\theta}_{b, j}$ are the corresponding orthonormal eigenvectors of $\mathbf{A}$ and $\mathbf{B}$. Then there exist two real orthogonal transformations $\boldsymbol{\Theta}_{a}=\left(\boldsymbol{\theta}_{a, 1}, \ldots, \boldsymbol{\theta}_{a, N}\right)\left(\boldsymbol{\Theta}_{a}^{T} \boldsymbol{\Theta}_{a}=\mathbf{I}_{N}\right)$ and $\boldsymbol{\Theta}_{b}=\left(\boldsymbol{\theta}_{b, 1}, \ldots, \boldsymbol{\theta}_{b, N}\right)\left(\boldsymbol{\Theta}_{b}^{T} \boldsymbol{\Theta}_{b}=\mathbf{I}_{N}\right)$, such that $\boldsymbol{\Theta}_{a}^{T} \mathbf{A} \boldsymbol{\Theta}_{a}=\mathbf{D}_{a}=\operatorname{diag}\left(\lambda_{a, j}\right)$ and $\boldsymbol{\Theta}_{b}^{T} \mathbf{B} \boldsymbol{\Theta}_{b}=\mathbf{D}_{b}=\operatorname{diag}\left(\lambda_{b, j}\right)$, respectively. Hence, the conventionally used real and non-negative coefficients $\omega_{j}$ and $\xi_{j}$ are derived

$$
\omega_{j}^{2}:=\overline{\mathbf{v}}_{j}^{T} \mathbf{B} \mathbf{v}_{j}=\left(\overline{\mathbf{v}}_{j}^{T} \mathbf{\Theta}_{b} \mathbf{D}_{b}^{1 / 2}\right)\left(\mathbf{D}_{b}^{1 / 2} \boldsymbol{\Theta}_{b}^{T} \mathbf{v}_{j}\right)
$$

and

$$
2 \xi_{j} \omega_{j}:=\overline{\mathbf{v}}_{j}^{T} \mathbf{A} \mathbf{v}_{j}=\left(\overline{\mathbf{v}}_{j}^{T} \mathbf{\Theta}_{a} \mathbf{D}_{a}^{1 / 2}\right)\left(\mathbf{D}_{a}^{1 / 2} \boldsymbol{\Theta}_{a}^{T} \mathbf{v}_{j}\right)
$$

$\omega_{j}^{2}$ and $2 \xi_{j} \omega_{j}$ can be interpreted as the squared norm of a complex vector and they depend on all eigenvalues $\lambda_{b, j}$ and $\lambda_{a, j}$, respectively. Hence, (A.2) becomes equivalent to Eq. (8). The elements of $\mathbf{C}$ (and $\mathbf{A}$ ) are assumed not to be too large so that all $\xi_{j}$ are less than 1 (underdamped system) and the eigenvalues $s_{j}$, solutions of (8), occur in complex conjugate pairs (see Eq.

(9)). The same property holds for the corresponding eigenvectors $\mathbf{v}_{j}$ as well as for $\phi_{j}$ and $\boldsymbol{\psi}_{j}$.

\section{A.1.2 The orthogonality conditions in the state space}

By definition, the characteristic pair $\left(s_{j}, \boldsymbol{\psi}_{j}\right)$ fulfils the relationship

$$
s_{j} \mathcal{A} \psi_{j}=-\mathcal{B} \psi_{j}
$$

Eq. (A.3) also holds for another characteristic pair $\left(s_{i}, \boldsymbol{\psi}_{i}\right)$, with $s_{i} \neq s_{j}$ :

$$
s_{i} \mathcal{A} \psi_{i}=-\mathcal{B} \psi_{i}
$$

Pre-multiplying (A.3) by $\boldsymbol{\psi}_{i}^{T}$ and (A.4) by $\boldsymbol{\psi}_{j}^{T}$, one obtains

$$
s_{j} \boldsymbol{\psi}_{i}^{T} \mathcal{A} \boldsymbol{\psi}_{j}=-\boldsymbol{\psi}_{i}^{T} \mathcal{B} \boldsymbol{\psi}_{j} \quad \text { and } \quad s_{i} \boldsymbol{\psi}_{j}^{T} \quad \mathcal{A} \boldsymbol{\psi}_{i}=-\boldsymbol{\psi}_{j}^{T} \mathcal{B} \boldsymbol{\psi}_{i}
$$

Then, the symmetry of $\mathcal{A}$ and $\mathcal{B}$ leads to the orthogonality conditions

$$
\boldsymbol{\psi}_{i}^{T} \mathcal{A} \boldsymbol{\psi}_{j}=0, \quad \boldsymbol{\psi}_{i}^{T} \mathcal{B} \boldsymbol{\psi}_{j}=0 \quad \text { for } s_{j} \neq s_{i} \text { and } i, j=1,2 N
$$


Using (3) and (6), it is straightforward to prove that (A.5) is equivalent to

$$
\left\{\begin{array}{l}
\boldsymbol{\phi}_{i}^{T} \mathbf{C} \boldsymbol{\phi}_{j}+\left(s_{i}+s_{j}\right) \boldsymbol{\phi}_{i}^{T} \mathbf{M} \boldsymbol{\phi}_{j}=0 \\
\boldsymbol{\phi}_{i}^{T} \mathbf{K} \boldsymbol{\phi}_{j}-s_{i} s_{j} \boldsymbol{\phi}_{i}^{T} \mathbf{M} \boldsymbol{\phi}_{j}=0
\end{array} \quad \text { for } s_{j} \neq s_{i}, \quad i, j=1,2 N\right.
$$

When $s_{j}=\bar{s}_{i}$, the above relationships are still valid. Hence, substituting Eq. (10) in Eq. (A.6) gives

$$
\left\{\begin{array}{l}
\boldsymbol{\phi}_{i}^{T} \mathbf{C} \overline{\boldsymbol{\phi}}_{i}-2 \xi_{i} \omega_{i} \boldsymbol{\phi}_{i}^{T} \mathbf{M} \overline{\boldsymbol{\phi}}_{i}=0 \\
\boldsymbol{\phi}_{i}^{T} \mathbf{K} \overline{\boldsymbol{\phi}}_{i}-\omega_{i}^{2} \boldsymbol{\phi}_{i}^{T} \mathbf{M} \overline{\boldsymbol{\phi}}_{i}=0 \quad \text { for } \quad i=1, N
\end{array}\right.
$$

which is similar to the conventional expression for systems having real modes.

\section{A.1.3 The particular case of real modes}

This section considers the case where the eigenvectors of the system (1) are real and the transformation associated with them produces a system of $N$ uncoupled equations. The so-called proportional damping condition, which implies that eigenvectors are real, is reviewed.

In light of the normalization introduced in Section A.1.1, the problem is reduced to finding conditions which ensure that the eigenvectors $\mathbf{v}_{i}$ in (A.1) are real, orthogonal and simultaneously diagonalize matrices $\mathbf{A}$ and $\mathbf{B}$. Provided that $\mathbf{A}$ and $\mathbf{B}$ are independently diagonalizable, a real and orthogonal transformation $\mathbf{V}=\left(\mathbf{v}_{1}, \ldots, \mathbf{v}_{N}\right)$ that simultaneously diagonalizes both matrices exists if and only if $\mathbf{A B}=\mathbf{B A}$ (see e.g. [6]). The commutativity of this product can be expressed in terms of $\mathbf{M}, \mathbf{C}, \mathbf{K}$ matrices and leads to the so-called proportional or classical damping condition:

$$
\left(\mathbf{M}^{-1} \mathbf{C}\right)\left(\mathbf{M}^{-1} \mathbf{K}\right)=\left(\mathbf{M}^{-1} \mathbf{K}\right)\left(\mathbf{M}^{-1} \mathbf{C}\right)
$$

One can also prove that $\mathbf{V}=\boldsymbol{\Theta}_{a}=\boldsymbol{\Theta}_{b}$. Let $\boldsymbol{\Phi}=\left(\boldsymbol{\varphi}_{1}, \ldots, \boldsymbol{\varphi}_{N}\right)=\mathbf{T} \mathbf{V}$ be the matrix of real eigenvectors $\boldsymbol{\varphi}_{j}$ (denoted by $\boldsymbol{\phi}_{j}$ in the general case). Hence, as in Eqs. (A.6) for the general case, the orthogonality conditions for a system having real modes can be derived

$$
\begin{aligned}
& \boldsymbol{\Phi}^{T} \mathbf{M} \boldsymbol{\Phi}=\mathbf{V}^{T} \mathbf{V}=\mathbf{I} \\
& \boldsymbol{\Phi}^{T} \mathbf{C} \boldsymbol{\Phi}=\mathbf{V}^{T} \mathbf{A V}=\operatorname{diag}\left(\lambda_{a, j}\right):=\operatorname{diag}\left(2 \xi_{j} \omega_{0 j}\right) \\
& \boldsymbol{\Phi}^{T} \mathbf{K} \boldsymbol{\Phi}=\mathbf{V}^{T} \mathbf{B V}=\operatorname{diag}\left(\lambda_{b, j}\right):=\operatorname{diag}\left(\omega_{0 j}^{2}\right)
\end{aligned}
$$


where $\lambda_{a, j}$ and $\lambda_{b, j}$ are the non-negative eigenvalues of $\mathbf{A}$ and $\mathbf{B}$ respectively. The first of the above relationships shows that $\boldsymbol{\varphi}_{j}$ are mass-normalized eigenvectors of the system (1). Moreover, in the special case when $\phi=\boldsymbol{\varphi}_{j}$, pre-multiplying Eq. (7) by $\varphi_{j}^{T}$ and making use of (A.9), leads to a system of $N$ uncoupled equations of the following type

$$
s_{j}^{2}+2 \xi_{j} \omega_{0 j} s_{j}+\omega_{0 j}^{2}=0
$$

where $\omega_{0 j}$ is the $\mathbf{j}$-th eigen circular frequency of the system (1) when $\mathbf{C}=\mathbf{0}$. It can be noted that $\omega_{0 j}$ only depends on the corresponding value $\lambda_{b, j}$, while, as already pointed out, in the general case $\omega_{j}$ is related to all values $\lambda_{b, i}, i=1, \ldots, j, \ldots, N$.

\section{A.2 Properties of the mother wavelet $\psi(t)$}

The mother wavelet $\psi(t)$ is said to be admissible if and only if $C_{\psi}=\int_{0}^{+\infty}|\hat{\psi}(a \omega)|^{2} \frac{d a}{a}$ is finite, non-zero and independent of the real number $\omega$ (see, for example [21]). This property allows the reconstruction of a signal from its wavelet transform, i.e. the inverse wavelet transform (ICWT) exists and is defined as follows:

$$
u(t)=\frac{1}{C_{\psi}} \int_{-\infty}^{+\infty} \int_{0}^{+\infty} T_{\psi}[u](b, a) \psi\left(\frac{t-b}{a}\right) \frac{d a}{a} d b
$$

The admissibility implies that the integral of $\psi(t)$ on the real axis is zero, since for $\omega=0$ the coefficient $C_{\psi}$ is finite only if $\hat{\psi}(0)=0$.

The mother wavelet is progressive when $\psi(t)$ belongs to the second Hardy space

$$
H^{2}(\mathbb{R})=\left\{\psi(t) \in L_{2}(\mathbb{R}): \hat{\psi}(\omega)=0 \text { for } \omega \leq 0\right\}
$$

This is equivalent to saying that the Fourier transform of the mother wavelet is zero for negative frequency values. Hence, progressive mother wavelets $\psi(t)$ are complex-valued. In addition, if $\hat{\psi}(\omega)$ is real, the real part of $\psi(t)$ is even, while the imaginary part is odd.

The concepts of time-frequency resolution and uncertainty coming from quantum mechanics are generally used to compare different mother wavelets $[21]$. Let $\tilde{\psi}(t)=\frac{|\psi(t)|^{2}}{\|\psi\|_{2}^{2}}$ and $\tilde{\hat{\psi}}(\omega)=\frac{|\hat{\psi}(\omega)|^{2}}{\|\hat{\psi}\|_{2}^{2}}$ be two real functions with unit 
integral, which means that they could be interpreted as probability distribution functions. Their expected values are

$$
t_{\psi}=\int_{-\infty}^{+\infty} t \frac{|\psi(t)|^{2}}{\|\psi\|_{2}^{2}} d t, \quad \omega_{\psi}=\int_{-\infty}^{+\infty} \omega \frac{|\hat{\psi}(\omega)|^{2}}{\|\hat{\psi}\|_{2}^{2}} d \omega
$$

If the Fourier transform of the mother wavelet $\psi(t)$ is real, then $\tilde{\psi}(t)$ is an even time-function and hence $t_{\psi}=0$. The standard deviations are respectively defined as

$$
\Delta t_{\psi}=\sqrt{\int_{-\infty}^{+\infty}\left(t-t_{\psi}\right)^{2} \frac{|\psi(t)|^{2}}{\|\psi\|_{2}^{2}} d t}, \quad \Delta \omega_{\psi}=\sqrt{\int_{-\infty}^{+\infty}\left(\omega-\omega_{\psi}\right)^{2} \frac{|\hat{\psi}(\omega)|^{2}}{\|\hat{\psi}\|_{2}^{2}}} d \omega(\mathrm{A}
$$

By definition, the smaller $\Delta t_{\psi}$, the larger the time-resolution of the mother wavelet (see Figure 2a). Conversely, the smaller $\Delta \omega_{\psi}$, the larger the frequency-resolution of $\psi$. However, the two resolutions cannot be both arbitrary large, since the Heisenberg uncertainty principle [21] states that the uncertainty $\mu_{\psi}$ is always greater than or equal to $\frac{1}{2}$

$$
\mu_{\psi}=\Delta t_{\psi} \Delta \omega_{\psi} \geq \frac{1}{2}
$$

Hence, a large time-resolution of the mother wavelet implies a small frequency resolution and viceversa.

By referring to (25) and (27), these notions are extended to the translated and scaled mother wavelet $\psi_{b, a}(t)=\frac{1}{a} \psi\left(\frac{t-b}{a}\right)$ and its Fourier transform $\hat{\psi}_{b, a}(\omega)=\hat{\psi}(a \omega) e^{-i b \omega}$. For mother wavelets with $t_{\psi}=0$, the expected values of $\tilde{\psi}_{b, a}(t)=\frac{\left|\psi_{b, a}(t)\right|^{2}}{\left\|\psi_{b, a}\right\|_{2}^{2}}$ and of $\tilde{\hat{\psi}}_{b, a}(\omega)=\frac{\left|\hat{\psi}_{b, a}(\omega)\right|^{2}}{\left\|\hat{\psi}_{b, a}\right\|_{2}^{2}}$ are

$$
t_{\psi_{b, a}}=b \quad \text { and } \quad \omega_{\psi_{b, a}}=\frac{\omega_{\psi}}{a}
$$

respectively (Figure $2 \mathrm{~b}$ ). The standard deviations are equal to

$$
\Delta t_{\psi_{b, a}}=a \Delta t_{\psi}, \quad \Delta \omega_{\psi_{b, a}}=\frac{\Delta \omega_{\psi}}{a}
$$

It can be said that $\psi_{b, a}$ is localized at the point $(b, a)$ with uncertainty $\mu_{\psi_{b, a}}=\Delta t_{\psi_{b, a}} \Delta \omega_{\psi_{b, a}}=\Delta t_{\psi} \Delta \omega_{\psi}=\mu_{\psi}$. Observe that $\mu_{\psi_{b, a}}$ is lower bounded 
independently from $(b, a)$, even if the single resolutions $\Delta t_{\psi_{b, a}}$ and $\Delta \omega_{\psi_{b, a}}$ depend on it. The second expression in (A.14) shows that a frequency value $\omega=\omega_{\psi_{b, a}}=\frac{\omega_{\psi}}{a}$ can be associated with each scale value $a$. For this reason, wavelet transform can also be used as a time-frequency representation.

\section{References}

[1] S. Adhikari, J. Woodhouse, Identification of Damping: Part 1, Viscous Damping, Journal of Sound and Vibration 243 (1) (2001) 43-61.

[2] S. Adhikari, J. Woodhouse, Identification of Damping: Part 2, Non-Viscous Damping, Journal of Sound and Vibration 243 (1) (2001) 63-88.

[3] V. Volterra, Sur la théorie mathématique des phénomènes héréditaires, Journal de Mathématiques Pures et Appliquées 7(3) (1928) 249-298 (in French).

[4] C.F. Beards, Structural Vibration, Analysis and damping, Arnold, London, 1996.

[5] J.W.S. Rayleigh, The Theory of Sound, 1st American edn., Dover Publications, New York, 1945.

[6] T.K. Caughey, Classical normal modes in damped linear dynamic systems, Journal of Applied Mechanics ASME (1960) 269-271.

[7] T.K. Caughey, M.E.J. Kelly, Classical normal modes in damped linear dynamic systems, Journal of Applied Mechanics ASME 52 (1965) 583-588.

[8] M. Géradin, D. Rixen, Théorie des Vibrations, Application à la Dynamique des Structures, 2nd Edition, Masson, Paris, 1996 (in French).

[9] E. Balmès, New results on the identification of normal modes from experimental complex modes, Mechanical Systems and Signal Processing 11(2) (1997) 229-243.

[10] S. Adhikari, Optimal complex modes and an index of damping non-proportionality, Mechanical Systems and Signal Processing 18 (1) (2004) $1-27$.

[11] N.M.M Maia, J.M.M. Silva, N. He, N.A. Lieven, R.M. Lin, G.W. Skingle, W.-M. To, A.P.V. Urgueira, Theoretical and Experimental Modal Analysis, Research Studies Press Ltd., Hertfordshire, 1998.

[12] M. Ruzzene, A. Fasana, L. Garibaldi, B. Piombo, Natural frequency and damping identification using wavelet transform: application to real data, Mechanical Systems and Signal Processing 11 (1997) 207-218.

[13] W.J. Stazewski, Identification of non-linear systems using multi-scale ridges and skeletons of the wavelet transform, Journal of Sound and Vibration 214(4) (1998) 639-658. 
[14] P. Argoul, T.P. Le, Instantaneous indicators of structural behaviour based on continuous Cauchy wavelet transform, Mechanical Systems and Signal Processing Journal 17 (2003) 243-250.

[15] T.P. Le, P. Argoul, Continuous wavelet transform for modal identification using free decay response, Journal of Sound and Vibration 277 (2004) 73-100.

[16] J. Slavič, I. Simonovski, M. Boltežar, Damping identification using a continuous wavelet transform: application to real data, Journal of Sound and Vibration 262 (2003) 291-307.

[17] J. Lardies, M.N. Ta, M. Berthillier, Modal parameter estimation based on the wavelet transform of output data, Archive of Applied Mechanics 73 (2004) 718-733.

[18] T.P. Le, Auscultation dynamique des structures l'aide de l'analyse continue en ondelettes, Ph.D. Thesis, ENPC, Paris (2003) (in French).

[19] A. Bhaskar, Taussky's theorem, symmetrizability and modal analysis revisited, Proceedings of the Royal Society London 457 (2001) 2455-2480.

[20] A.S. Veletsos, C.E. Ventura, Modal analysis of non-classically damped linear systems, Earthquake Engineering and Structural Dynamics 14 (1986) 217-243.

[21] R. Carmona, W.-L. Hwang, B. Torrésani, Practical Time-Frequency Analysis, Series: Wavelet Analysis and its Applications 9, Academic Press, San Diego, USA, 1998.

[22] C.K. Chui, An Introduction to Wavelets, Series: Wavelet Analysis and its Applications 1, Academic Press, Boston, USA, 1992.

[23] B. Torrésani, Analyse Continue par Ondelettes, Series: Savoir actuels, InterEditions / CNRS Editions, Paris, France, 1995, in French.

[24] M.N. Ta, Analyse modale par sous-espaces et par la transforme en ondelettes, Ph.D. Thesis, Université de Franche-Comté, Besançon (2005) (in French).

[25] W.J. Staszewski, Identification of damping in MDOF systems using time-scale decomposition, Journal of Sound and Vibration 203 (1997) 283-305.

[26] N. Delprat, B. Escudie, P. Guillemain, R.K. Martinet, Ph. Tchamitchian, B. Torresani, Asymptotic wavelet and Gabor analysis: extraction of instantaneous frequencies (special issue on Wavelet and Multiresolution Analysis), IEEE Transactions on Information Theory 38 (1992) 644-664.

[27] M. Boltezar and J. Slavic, Enhancements to the continuous wavelet transform for damping identifications on short signals, Mechanical Systems and Signal Processing 18(5) (2004) 1065-1076.

[28] P.A.P. Moran, An introduction of Probability theory, Oxford, 1968.

[29] F. Jaillet, B. Torrésani, Remarques sur l'adaptivité des représentations temps-fréquence, Proceedings of the GRETSI'03 conference, Paris (2003) (in French). 


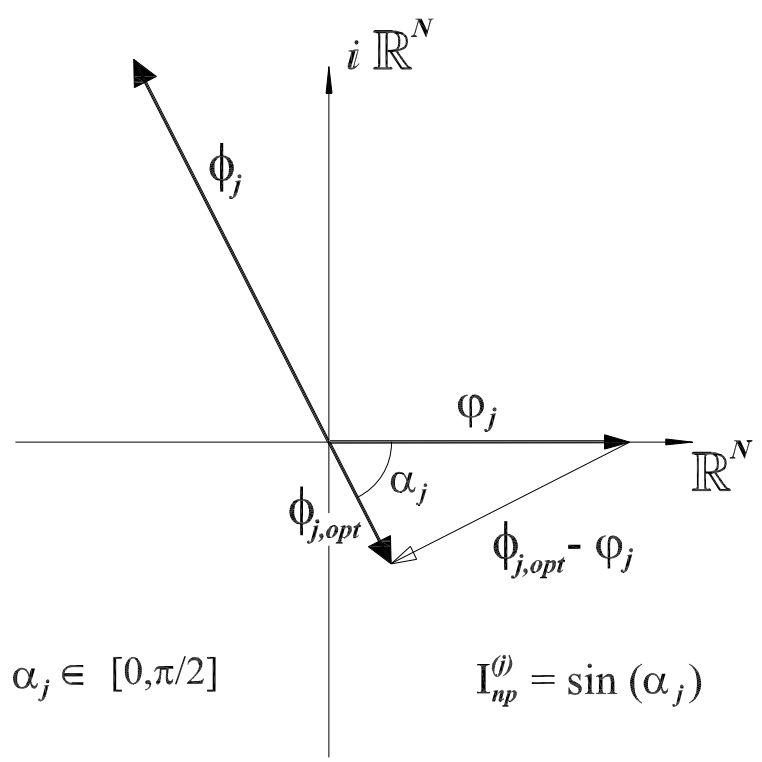

Fig. 1. Optimal complex mode shape and non-proportionality index. 


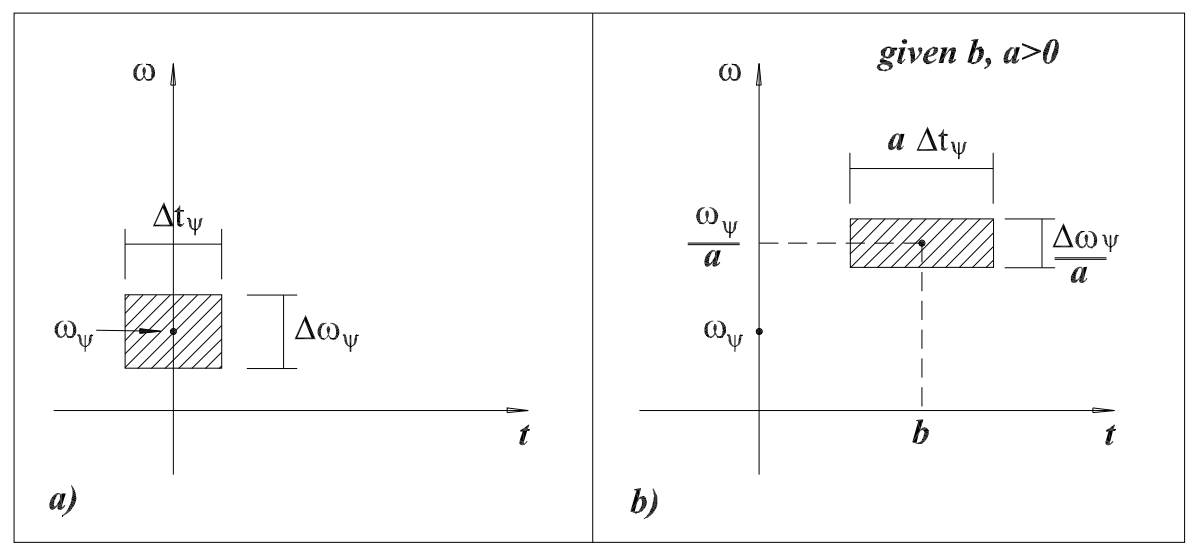

Fig. 2. Time-frequency resolution of: a) the mother wavelet $\psi(t)$ and of b) the translated and scaled mother wavelet $\psi_{b, a}(t)$. 


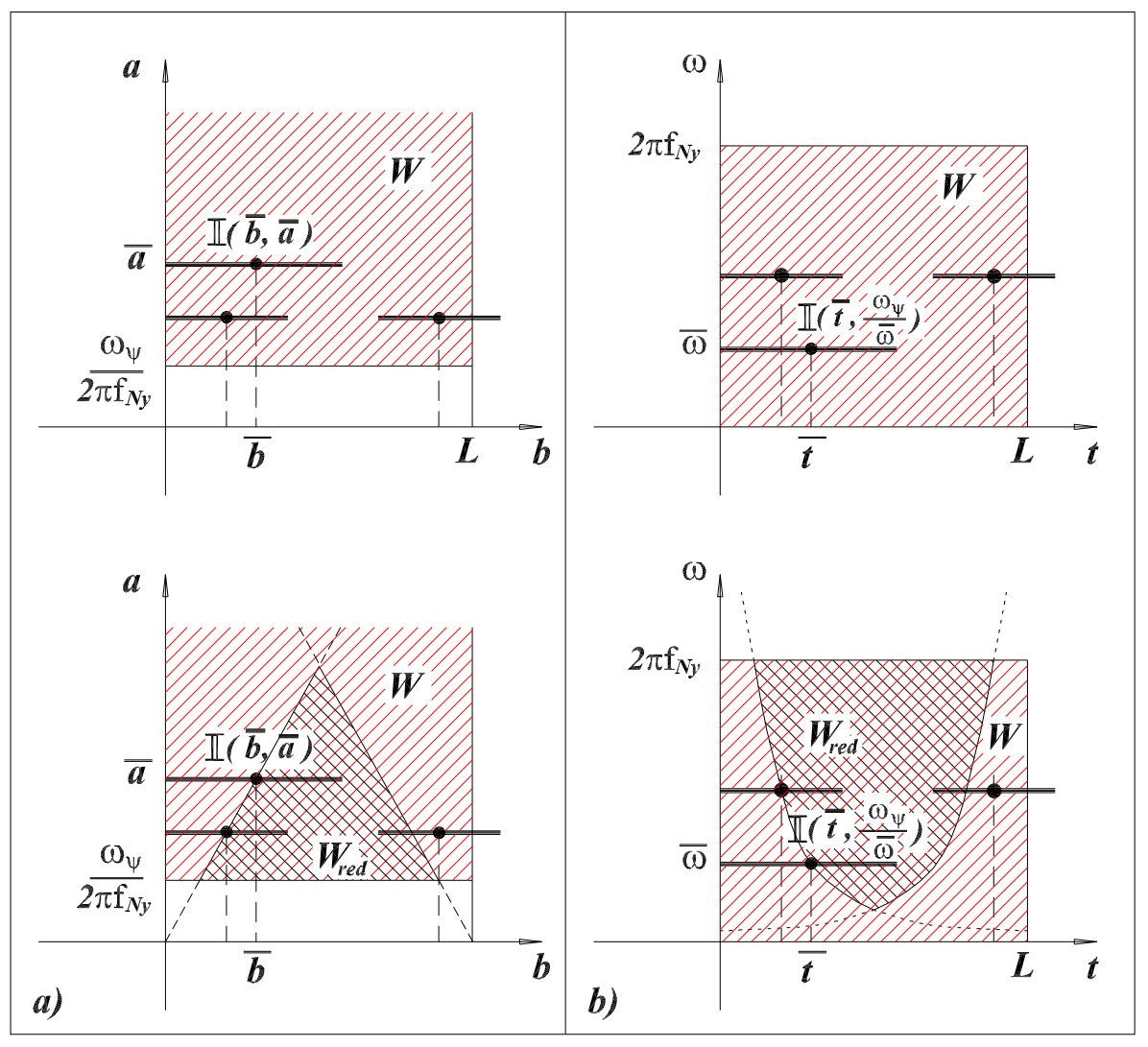

Fig. 3. a) Time-scale domains of the CWT and edge-effects; b) Time-frequency domains and edge-effects. 

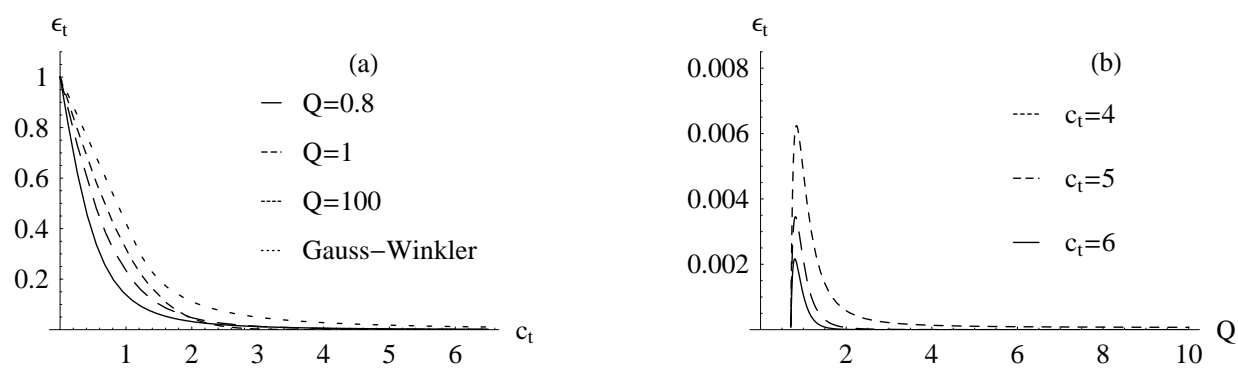

Fig. 4. (a) $\varepsilon_{t}$ vs. $c_{t}$ for the Cauchy mother wavelet with three Q-values. The upper curve corresponds to the two branches of the Gauss-Winkler inequality. (b) $\varepsilon_{t}$ vs. $Q$ for the Cauchy mother wavelet and for three $c_{t}$ values. Observe that $\varepsilon_{t}$ rapidly tends to zero for increasing $Q$. 


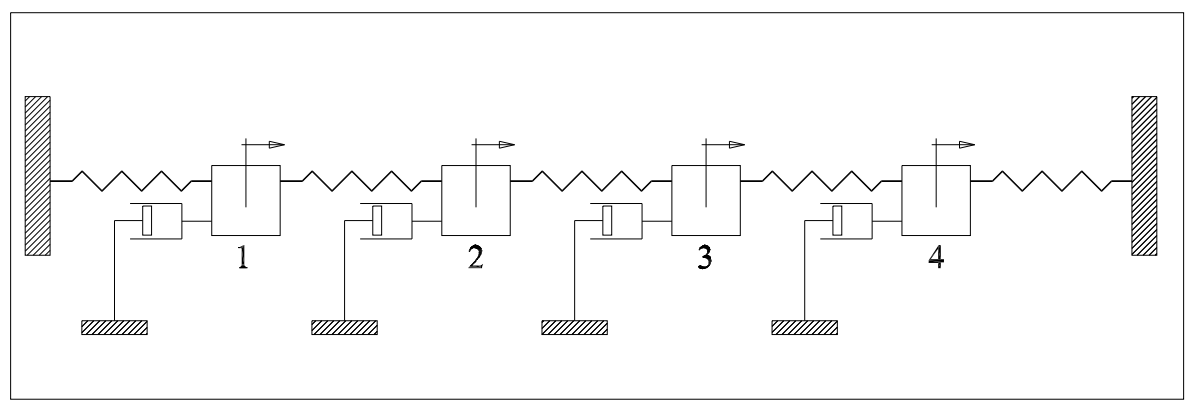

Fig. 5. The four DoF system of the numerical simulations. 
(a)

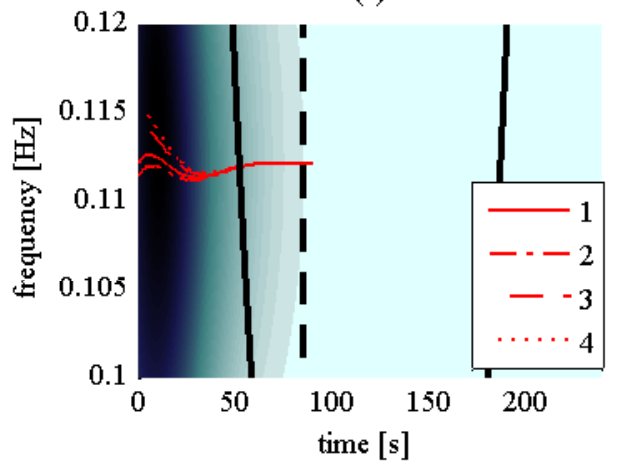

(b)

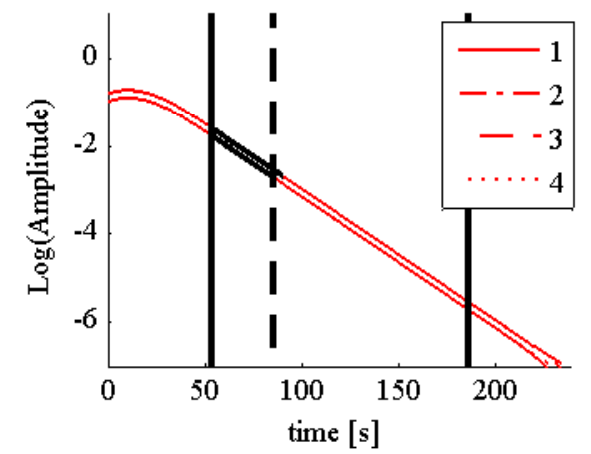

(a)

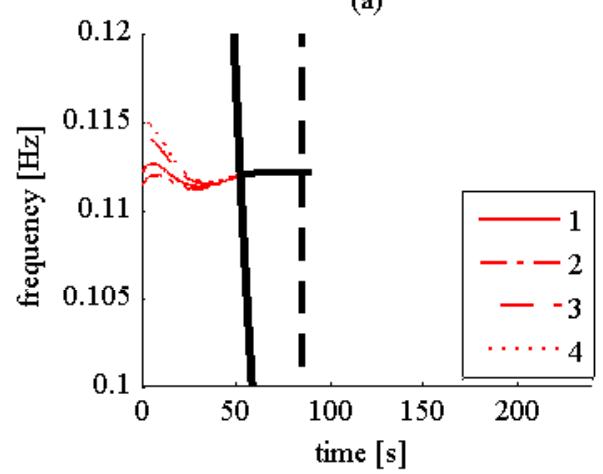

(c)

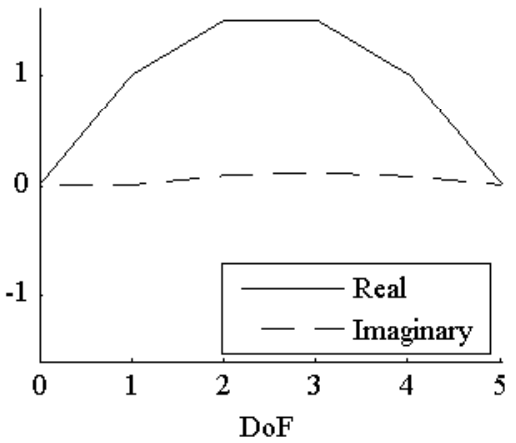

Fig. 6. First mode of system 1. a) CWT of signal 4 (contour plot) and ridges extracted from the four recorded signals up to the stopping time $t_{\bar{U}_{1}}$. b) Extracted ridges up to the stopping time $t_{\bar{U}_{1}}$. The effective part of the ridges is highlighted by the increased thickness of the lines. c) Logarithmic plot of the skeleton amplitudes. The effective part of the skeletons is highlighted by the increased thickness of the lines. d) Identified complex mode shape. In a), b) and c), the continuous hyperbolic and vertical thick lines delimit the edge effect region, like in the scheme of Figure $3 \mathrm{~b}$. The stopping time $t_{\bar{U}_{1}}$ is indicated by the vertical thick dashed line. 

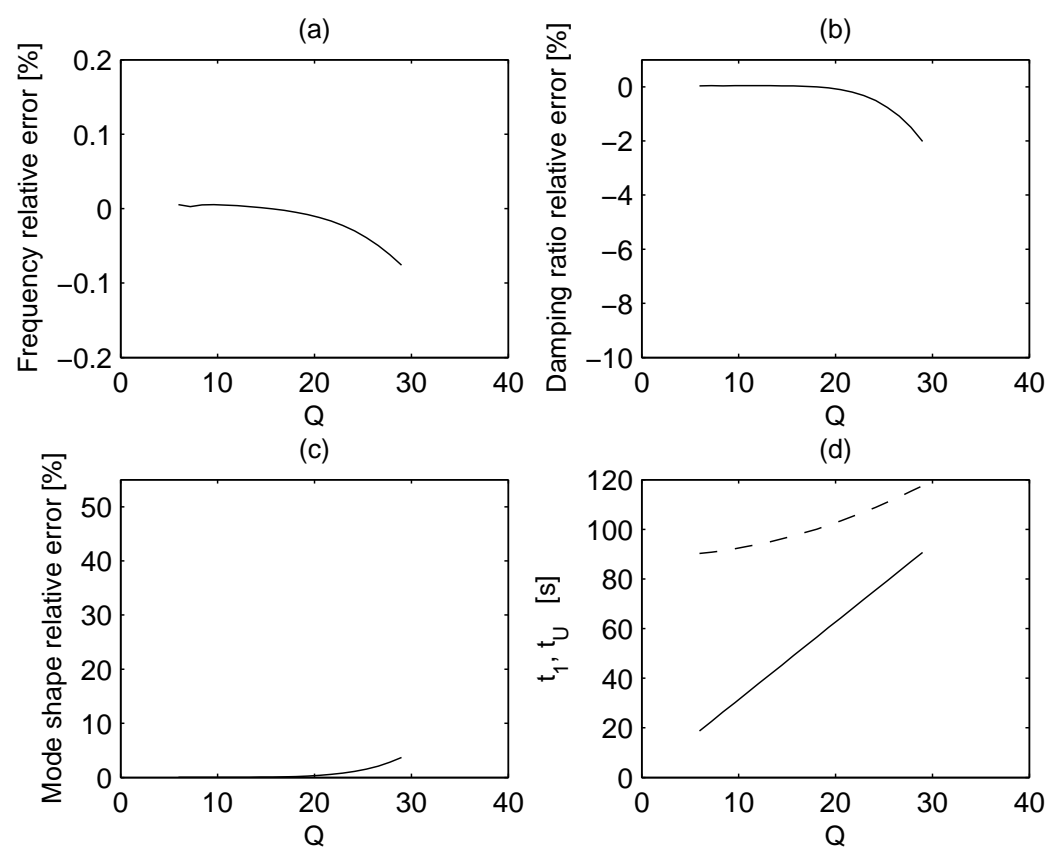

Fig. 7. Second mode of system 1. Relative errors vs. Q-factor. a) Modal frequency relative error; b) Damping ratio relative error; c) Mode shape relative error; d) Initial and stopping time of the effective part of the ridge. Note that the CWT analysis has been performed with $Q=11.7$, equal to the damping-related value $Q_{\xi}$. 

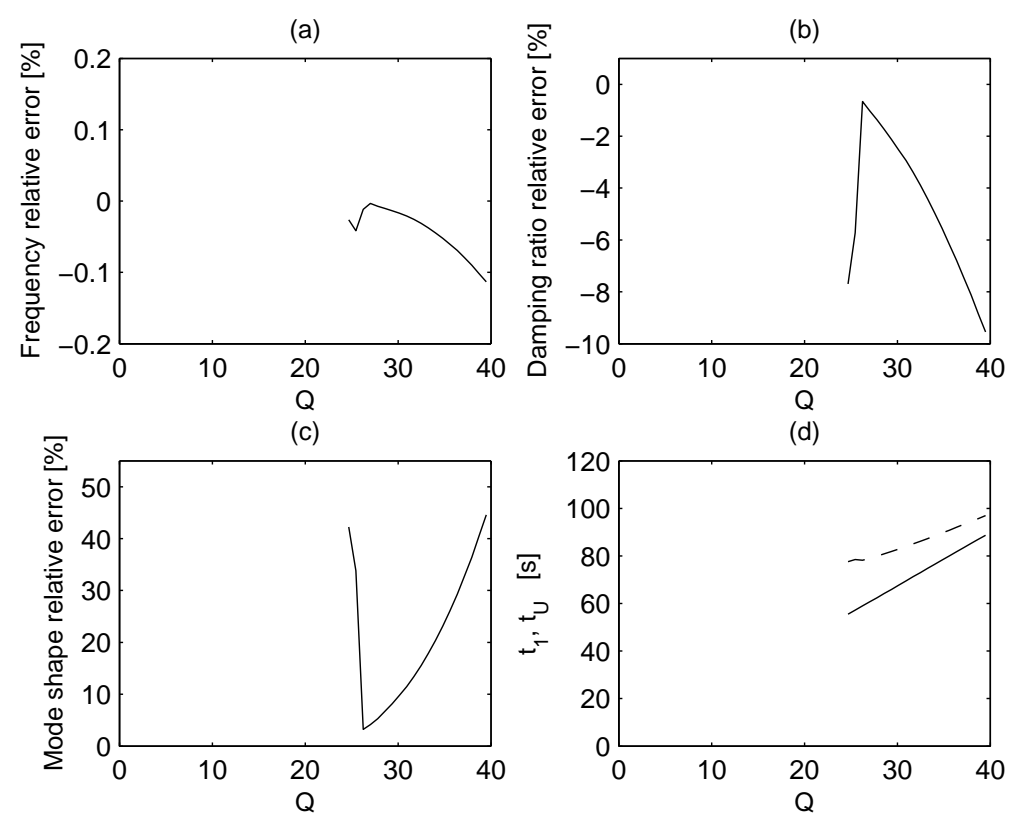

Fig. 8. Third mode of system 1. Relative errors vs. Q-factor. a) Modal frequency relative error; b) Damping ratio relative error; c) Mode shape relative error; d) Initial and stopping time of the effective part of the ridge. Note that the CWT analysis has been performed with $Q=Q_{\min }=26.9$. 
(a)

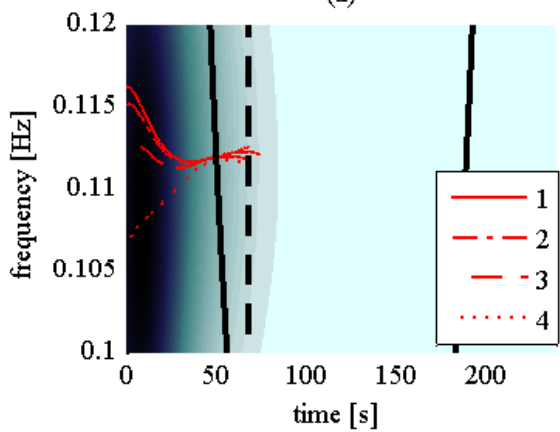

(b)

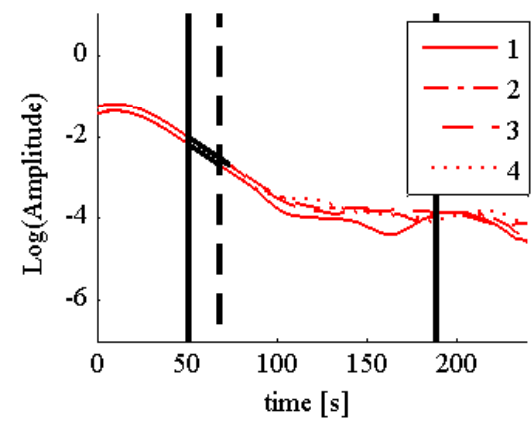

(a)

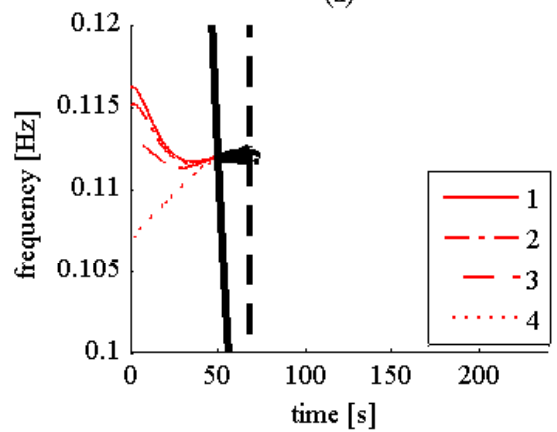

(c)

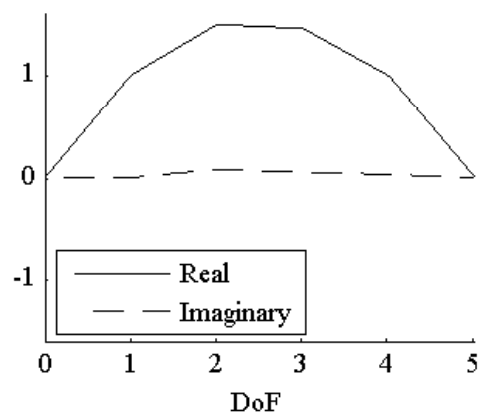

Fig. 9. First mode of system 1 with added noise. a) CWT of signal 4 (contour plot) and ridges extracted from the four recorded signals up to the stopping time $t_{\bar{U}_{1}}$. b) Ridges extracted from the four recorded signals up to the stopping time $t_{\bar{U}_{1}}$. Note that the threshold $\bar{U}_{1}$ and the stopping time are different with respect to the ones of the noise-free case. c) Logarithmic plot of the skeleton amplitudes. Note the non-decreasing skeleton amplitude after the stopping time, due to the added noise. d) Identified complex mode shape. The continuous and dashed thick lines have the same meaning as in Figure 6. 

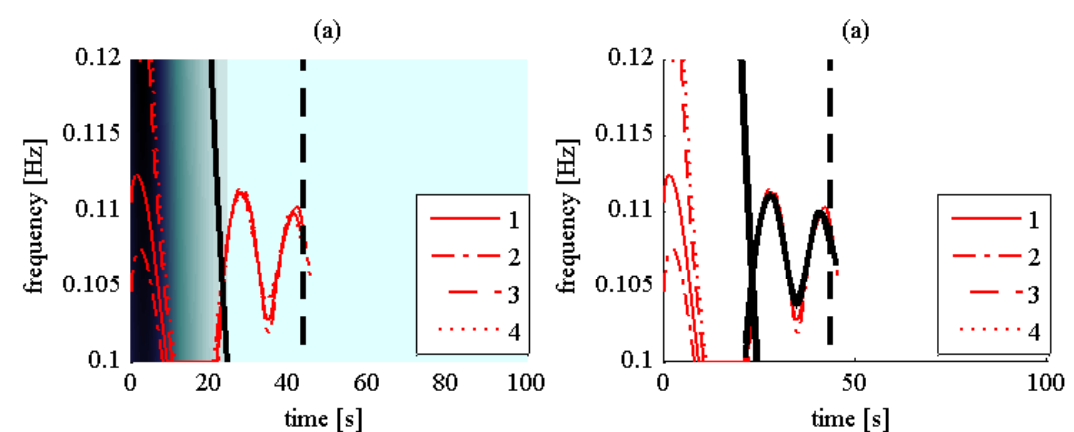

(b)
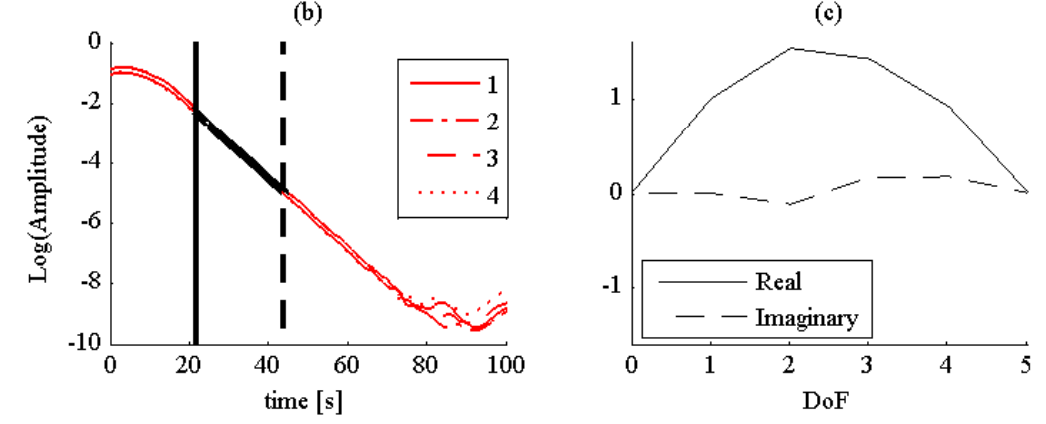

Fig. 10. First mode of system 2. a) CWT of signal 4 (contour plot) and ridges extracted from the four recorded signals up to the stopping time $t_{\bar{U}_{1}}$. b) Ridges extracted from the four recorded signals up to the stopping time $t_{\bar{U}_{1}}$. c) Logarithmic plot of the skeleton amplitudes. d) Identified complex mode shape. The continuous and dashed thick lines have the same meaning as in previous Figures. 

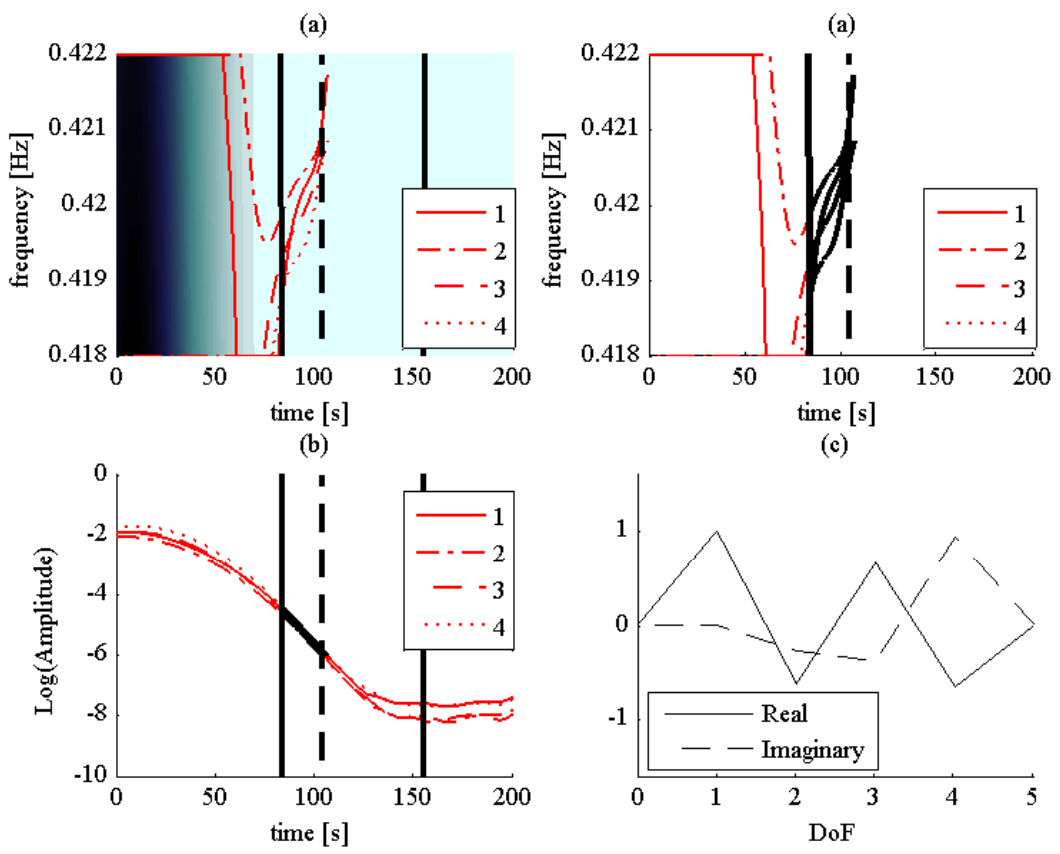

(c)

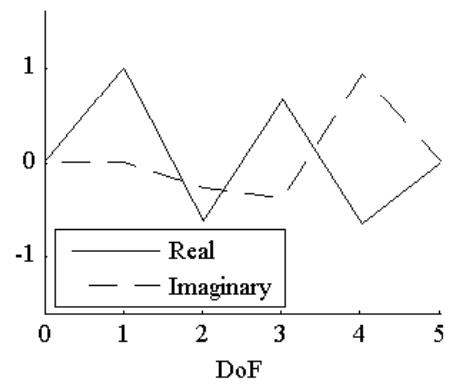

Fig. 11. Fourth mode of system 2. a) CWT of signal 4 (contour plot) and ridges extracted from the four recorded signals up to the stopping time $t_{\bar{U}_{4}}$. b) Ridges extracted from the four recorded signals up to the stopping time $t_{\bar{U}_{4}}$. c) Logarithmic plot of the skeleton amplitudes. d) Identified complex mode shape. The continuous and dashed thick lines have the same meaning as in previous Figures. 

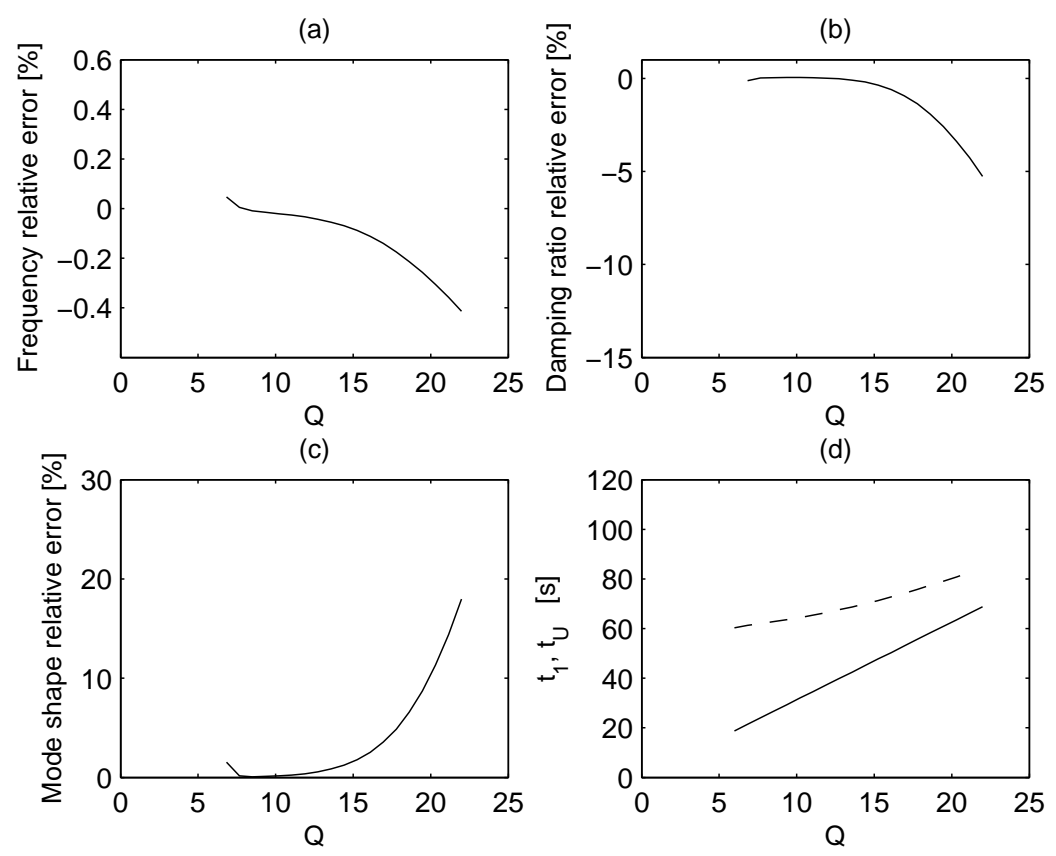

Fig. 12. Second mode of system 2. Relative errors vs. Q-factor. a) Modal frequency relative error; b) Damping ratio relative error; c) Mode shape relative error; d) Initial and stopping time of the effective part of the ridge. Note that the CWT analysis has been performed with $Q=7.8\left(Q_{\xi}=7.82\right.$ and $\left.Q_{\min }=7\right)$. 

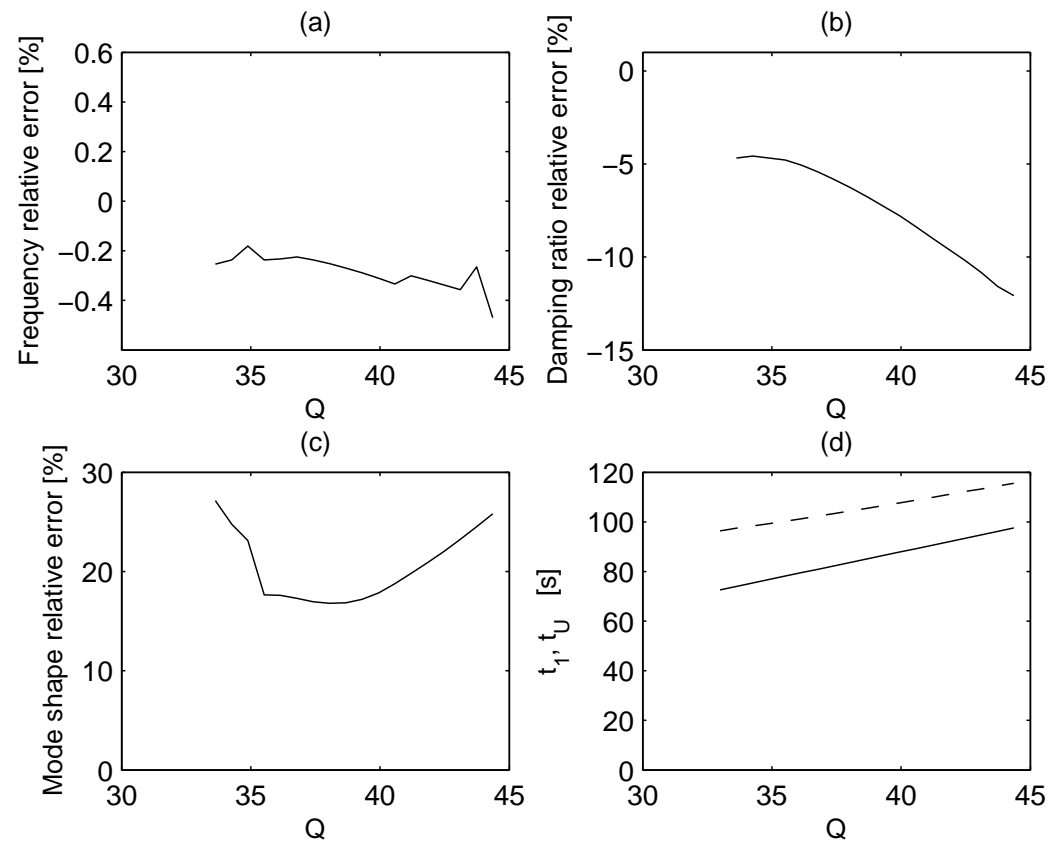

Fig. 13. Fourth mode of system 2. Relative errors vs. Q-factor. a) Modal frequency relative error; b) Damping ratio relative error; c) Mode shape relative error; d) Initial and stopping time of the effective part of the ridge. Note that the CWT analysis has been performed with $Q=Q_{\min }=38.5$. The errors remain quite large, since this mode is not excited enough by the shock applied on the second mass. 


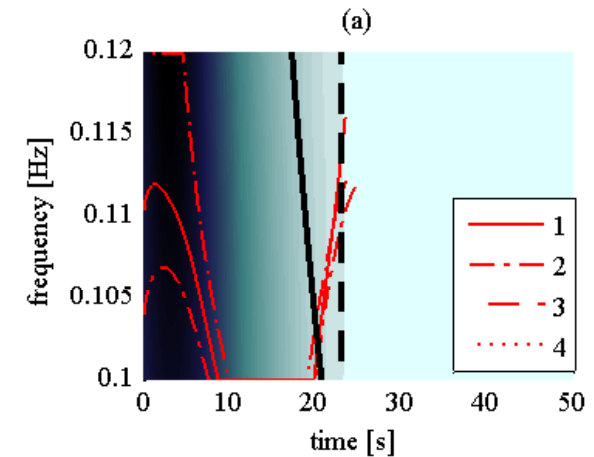

(b)

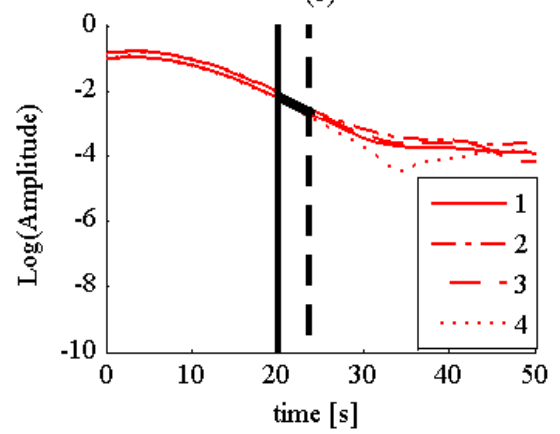

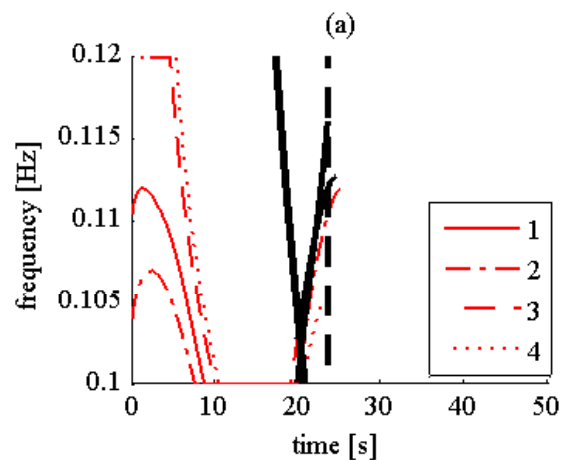

(c)

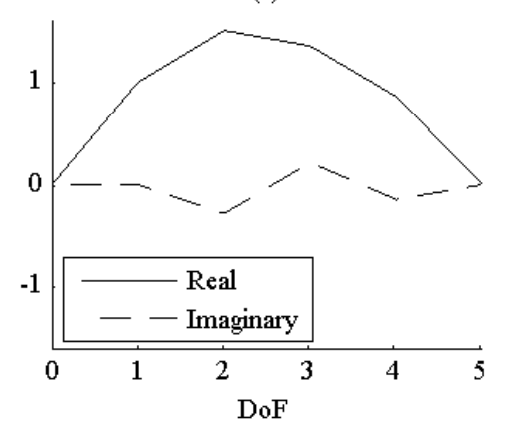

Fig. 14. First mode of system 2 with added noise. a) CWT of signal 4 (contour plot) and ridges extracted from the four recorded signals up to the stopping time $t_{\bar{U}_{1}}$. b) Ridges extracted from the four recorded signals up to the stopping time $t_{\bar{U}_{1}}$. Note that the threshold $\bar{U}_{1}$ and the stopping time are different with respect to the ones of the noise-free case. c) Logarithmic plot of the skeleton amplitudes. Note the non-decreasing skeleton amplitude after the stopping time, due to the added noise. d) Identified complex mode shape. The continuous and dashed thick lines have the same meaning as in the previous Figures. 


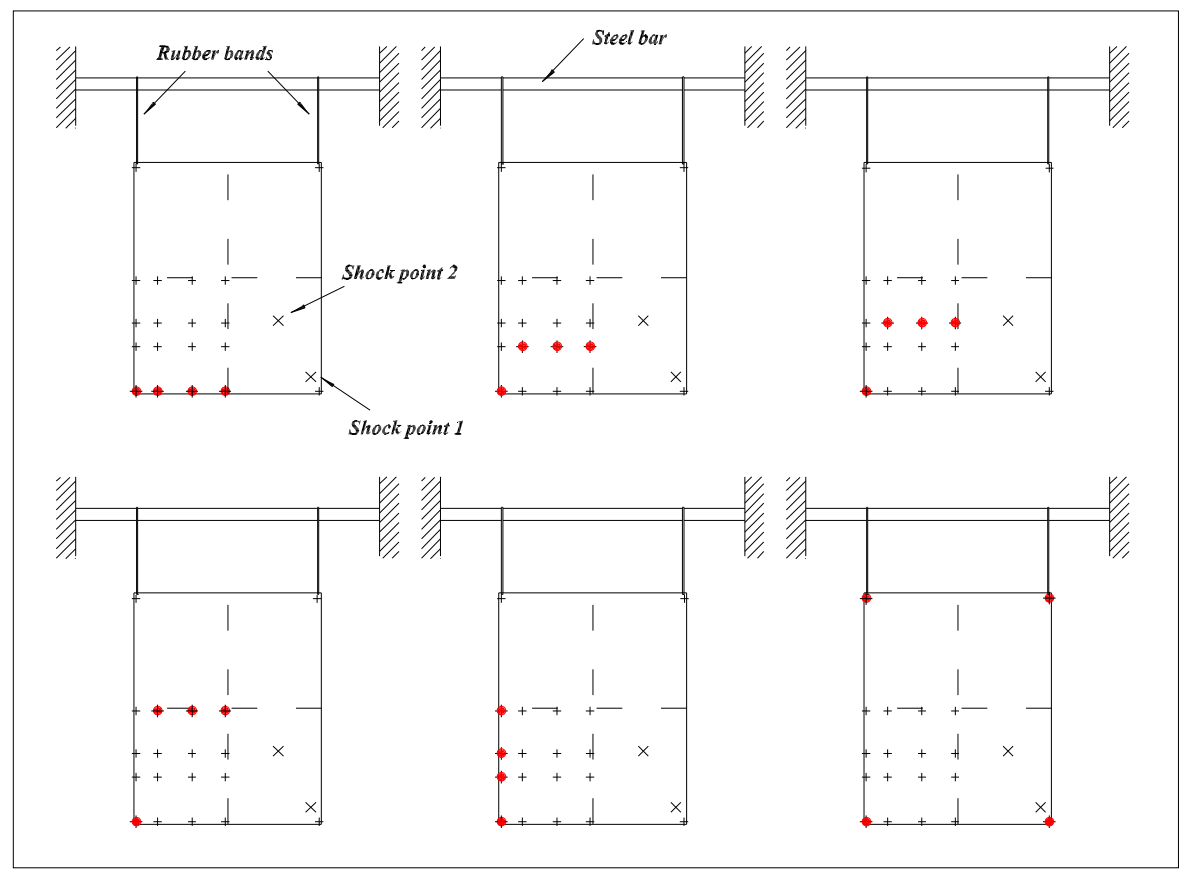

Fig. 15. Set-up for the plexiglass plate experiments with the six accelerometer locations. The symmetry of the plate has been exploited in order to reduce the number of recorded signals. 

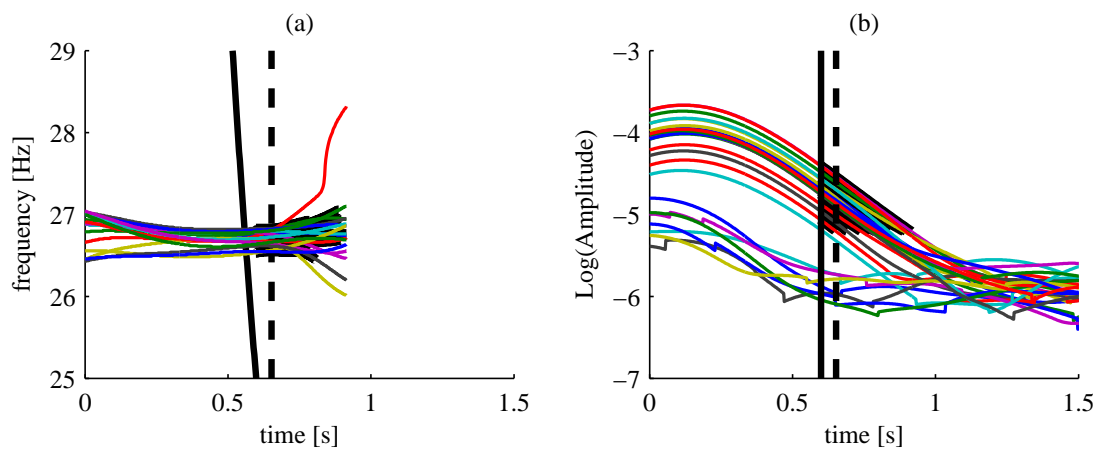

(c)

(d)
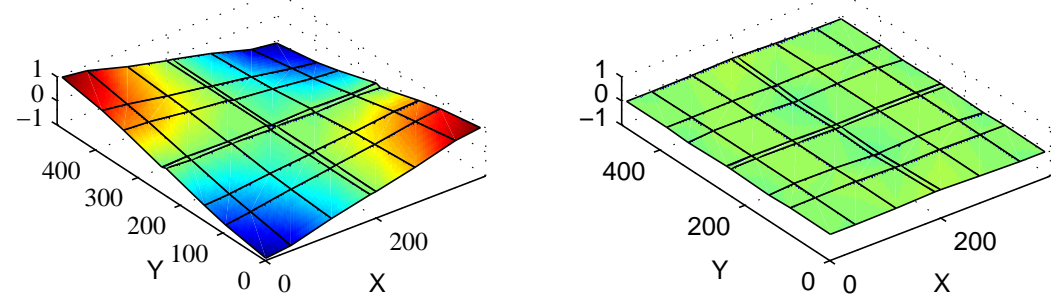

Fig. 16. Plexiglass plate, mode 1. a) Ridges extracted from the selected recorded signals. b) Logarithmic plot of the skeleton amplitudes, with the time intervals suitable for modal analysis. c) Real part of the identified complex mode shape. d) Imaginary part of the identified complex mode shape. 

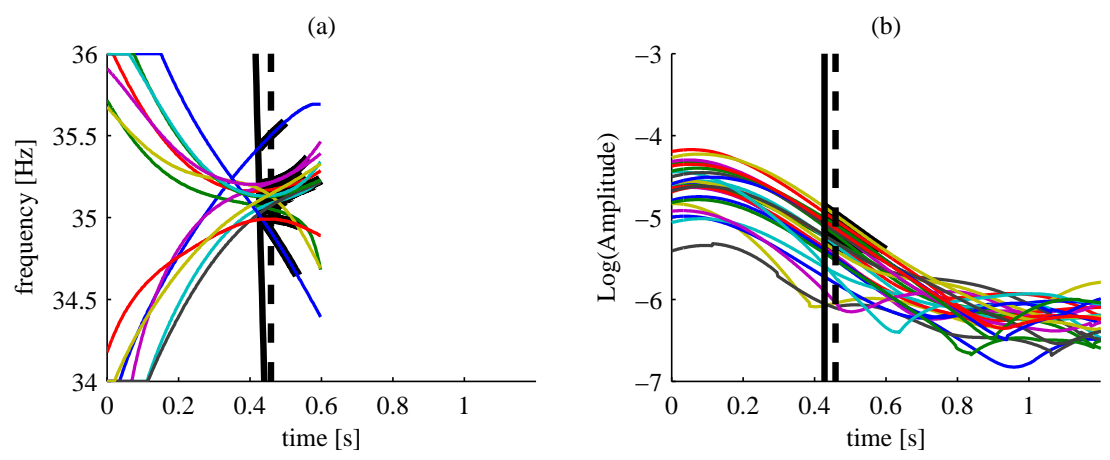

(c)

(d)
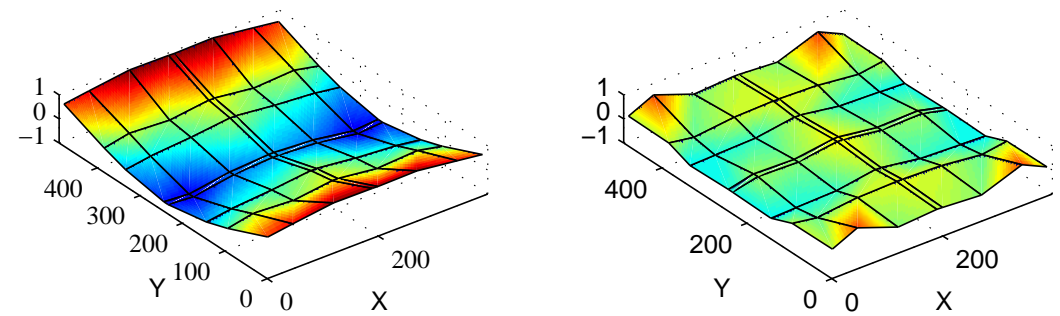

Fig. 17. Plexiglass plate, mode 2. a) Ridges extracted from the selected recorded signals. b) Logarithmic plot of the skeleton amplitudes, with the time intervals suitable for modal analysis. c) Real part of the identified complex mode shape. d) Imaginary part of the identified complex mode shape. 


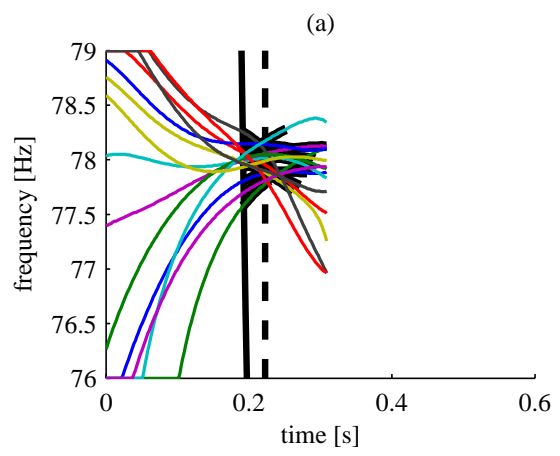

(c)

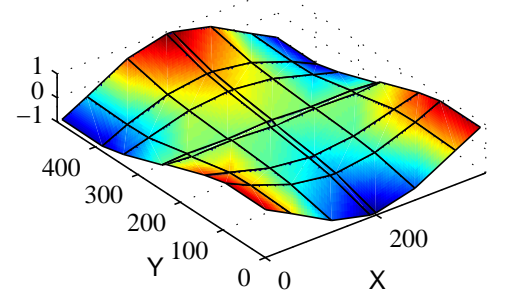

(b)

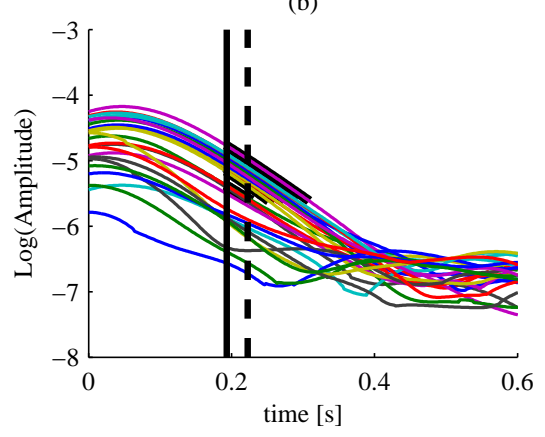

(d)

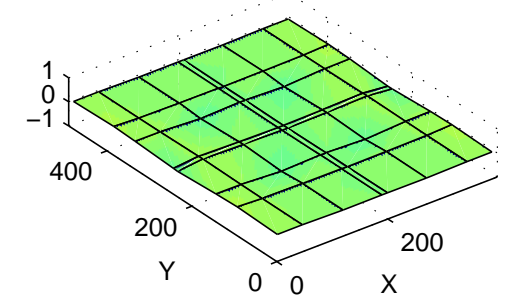

Fig. 18. Plexiglass plate, mode 5. a) Ridges extracted from the selected recorded signals. b) Logarithmic plot of the skeleton amplitudes, with the time intervals suitable for the modal analysis. c) Real part of the identified complex mode shape. d) Imaginary part of the identified complex mode shape. 
(a)

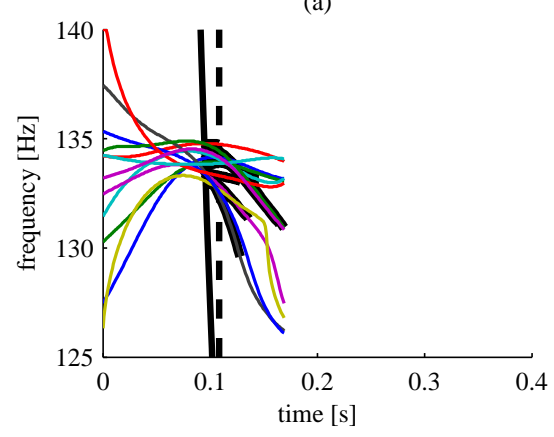

(c)

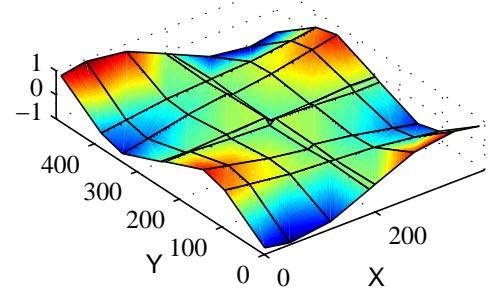

(b)

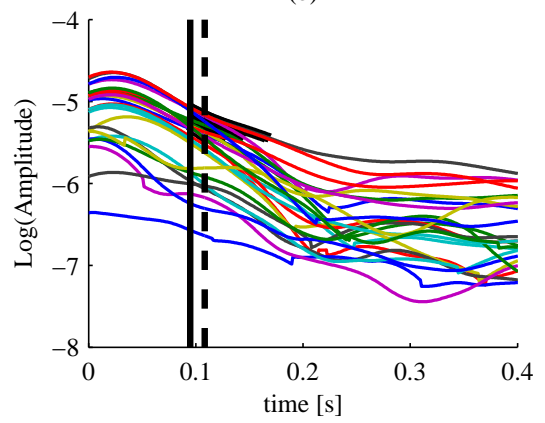

(d)

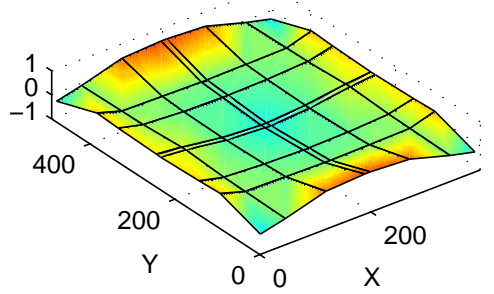

Fig. 19. Plexiglass plate, mode 7. a) Ridges extracted from the selected recorded signals. b) Logarithmic plot of the skeleton amplitudes, with the time intervals suitable for the modal analysis. c) Real part of the identified complex mode shape. d) Imaginary part of the identified complex mode shape. 
(a)

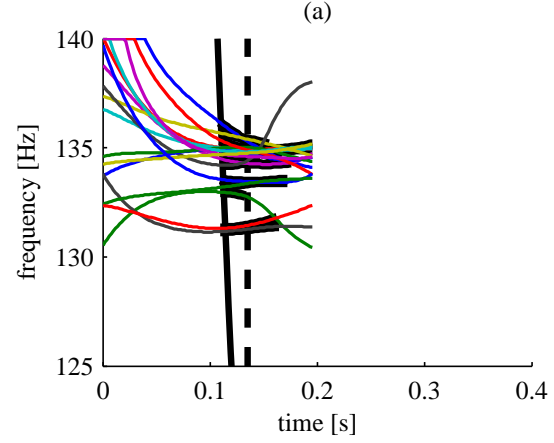

(c)

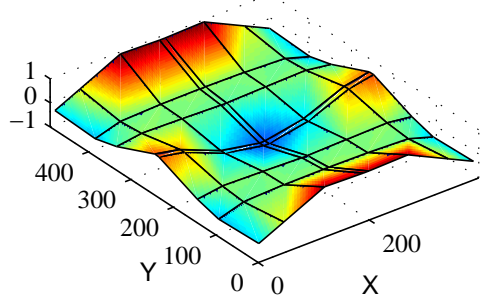

(b)

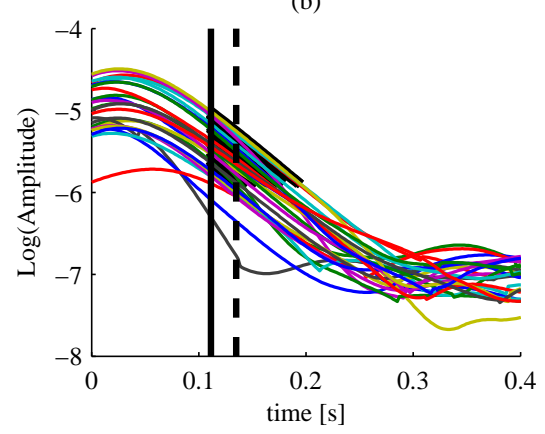

(d)

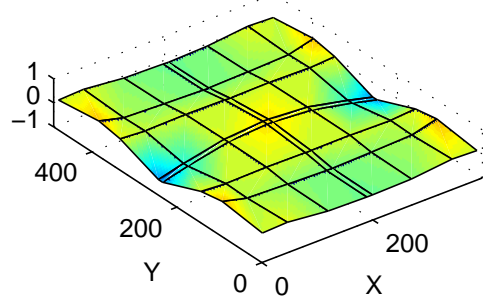

Fig. 20. Plexiglass plate, mode 8. a) Ridges extracted from the selected recorded signals. b) Logarithmic plot of the skeleton amplitudes, with the time intervals suitable for the modal analysis. c) Real part of the identified complex mode shape. d) Imaginary part of the identified complex mode shape. 
(a)

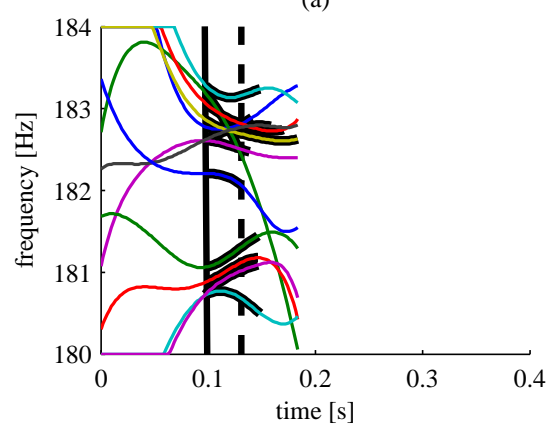

(c)

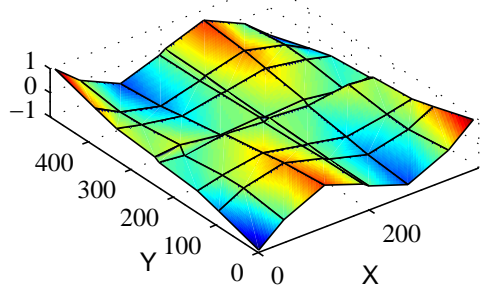

(b)

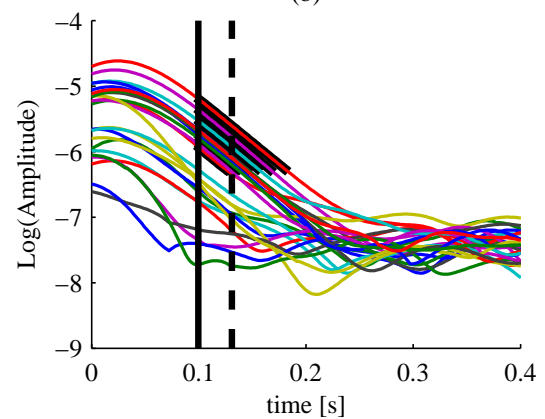

(d)

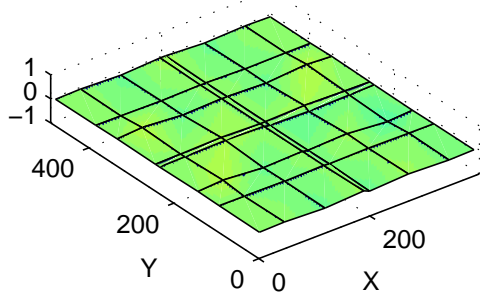

Fig. 21. Plexiglass plate, mode 10. a) Ridges extracted from the selected recorded signals. b) Logarithmic plot of the skeleton amplitudes, with the time intervals suitable for the modal analysis. c) Real part of the identified complex mode shape. d) Imaginary part of the identified complex mode shape. 


\begin{tabular}{ccccc}
\hline Mode $(\mathbf{j})$ & $\mathbf{1}$ & $\mathbf{2}$ & $\mathbf{3}$ & $\mathbf{4}$ \\
\hline Undamped freq. $f_{0 j}[\mathrm{~Hz}]$ & 0.1125 & 0.2809 & 0.3898 & 0.4325 \\
\hline Real mode shape $\boldsymbol{\varphi}_{j}$ & $\left(\begin{array}{c}1 \\
1.5000 \\
1.5000 \\
1\end{array}\right)$
\end{tabular}

Table 1

Modal properties of the undamped system defined by the mass and stiffness matrices of Eq. (61).

\begin{tabular}{|c|c|c|c|c|c|}
\hline $\begin{array}{c}\text { Mode } \\
\qquad(\mathrm{j})\end{array}$ & $\begin{array}{c}\tilde{f}_{j} \\
{[\mathrm{~Hz}]}\end{array}$ & $\begin{array}{c}\xi_{j} \\
{[\%]}\end{array}$ & $\phi_{j}$ & $I_{n p}^{(j)}$ & $\tilde{I}_{n p}^{(j)}$ \\
\hline 1 & 0.1122 & 9.65 & $\left(\begin{array}{cc}1 & \\
1.4912+i & 0.0854 \\
1.4886+i & 0.1088 \\
0.9917+i & 0.0782\end{array}\right)$ & 0.0258 & 0.0258 \\
\hline 2 & 0.2814 & 6.01 & $\begin{array}{c}1 \\
0.6109+i 0.1691 \\
-0.6184-i 0.0293 \\
-0.9733-i 0.1505\end{array}$ & 0.0960 & 0.0963 \\
\hline 3 & 0.3897 & 5.59 & $\begin{array}{c}1 \\
-0.3481+i 0.1842 \\
-0.2775-i 0.1551 \\
0.8805+i 0.0906\end{array}$ & 0.1891 & 0.1778 \\
\hline 4 & 0.4296 & 3.26 & $\begin{array}{c}1 \\
-0.7741+i 0.2914 \\
0.7456-i 0.4723 \\
-0.9151+i 0.7018\end{array}$ & 0.2715 & 0.2827 \\
\hline
\end{tabular}

Table 2

Modal properties of system 1, defined by the mass and stiffness matrices of Eq. (61) and the damping matrix (62), with $C_{11}=0.5$ and $C_{22}=0.2$. 


\begin{tabular}{ccccccccccc}
\hline $\begin{array}{c}\text { Mode } \\
(\mathbf{j})\end{array}$ & $c_{t}, c_{f}$ & $\begin{array}{c}\bar{U}_{j} \\
\tilde{f}_{j, \text { estim }} \\
{[\mathrm{m}]}\end{array}$ & $\begin{array}{c}\xi_{j, \text { estim }} \\
{[\mathrm{Hz}]}\end{array}$ & $\begin{array}{c}Q_{\min } \\
{[\%]}\end{array}$ & $Q_{\max }$ & $Q_{\xi}$ & $Q$ & $t_{1}$ & $t_{\bar{U}_{j}}$ \\
\hline $\mathbf{1}$ & 5.5 & $10^{-5}$ & 0.11 & 9.6 & 2.75 & 14.96 & $\mathbf{7 . 3 3}$ & $\mathbf{7 . 3}$ & 58.14 & 162.09 \\
$\mathbf{2}$ & 5.5 & $10^{-5}$ & 0.28 & 6.0 & 7.0 & 38.09 & $\mathbf{1 1 . 7 6}$ & $\mathbf{1 1 . 7}$ & 36.63 & 93.72 \\
$\mathbf{3}$ & 5.5 & $10^{-5}$ & 0.39 & 5.6 & $\mathbf{2 6 . 8 1}$ & 53.05 & 12.61 & $\mathbf{2 6 . 9}$ & 60.47 & 78.84 \\
$\mathbf{4}$ & 5.5 & $10^{-5}$ & 0.43 & 3.3 & $\mathbf{2 9 . 5 6}$ & 58.49 & 21.42 & $\mathbf{2 9 . 6}$ & 60.35 & 117.33 \\
\hline
\end{tabular}

Table 3

Parameters related to the choice of $Q$-factor for the CWT analysis of system 1.

\begin{tabular}{ccccc}
\hline $\begin{array}{c}\text { Mode } \\
(\mathbf{j})\end{array}$ & $\Delta t_{u}$ & $\Delta t_{\psi_{b, a}}$ & $d f_{\text {estim }}$ & $c_{f} \frac{\Delta \omega_{\psi_{b, a}}}{2 \pi}=c_{f} \frac{\tilde{f}_{j, \text { stim }}}{2 Q}$ \\
\hline $\mathbf{1}$ & $\mathbf{1 0 . 6 6}$ & $\mathbf{1 0 . 5 6}$ & 0.110 & 0.041 \\
$\mathbf{2}$ & $\mathbf{6 . 7 0}$ & $\mathbf{6 . 6 5}$ & 0.110 & 0.066 \\
$\mathbf{3}$ & 5.16 & 11.98 & $\mathbf{0 . 0 4 0}$ & $\mathbf{0 . 0 4 0}$ \\
$\mathbf{4}$ & 7.93 & 11.96 & $\mathbf{0 . 0 4 0}$ & $\mathbf{0 . 0 4 0}$ \\
\hline
\end{tabular}

Table 4

System 1. Characteristic time of the signal $\Delta t_{u}=\frac{1}{\sqrt{2}} \frac{1}{\xi_{j, e s t i m} 2 \pi \tilde{f}_{j, e s t i m}}$ and time standard deviation $\Delta t_{\psi_{b, a}}=\frac{1}{\sqrt{4 Q^{2}-2}} \frac{2 Q^{2}}{2 \pi \tilde{f}_{j, \text { estim }}}$ of the translated and scaled mother wavelet (columns 1 and 2); estimated frequency distance $d f_{\text {estim }}$ between close peaks and frequency standard deviation $\Delta \omega_{\psi_{b, a}}$ multiplied by the factor $c_{f}$ (columns 3 and $4)$. 


\begin{tabular}{|c|c|c|c|c|c|c|c|}
\hline $\begin{array}{c}\text { Mode } \\
\qquad(\mathrm{j})\end{array}$ & $\begin{array}{c}\tilde{f}_{j, \text { ident }} \\
{[\mathrm{Hz}]}\end{array}$ & $\begin{array}{c}\xi_{j, i d e n t} \\
{[\%]}\end{array}$ & $\phi_{j, i d e n t}$ & $\tilde{I}_{n p, i d e n t}^{(j)}$ & $\begin{array}{c}E r r_{f} \\
{[\%]}\end{array}$ & $\begin{array}{c}\operatorname{Err}_{\xi} \\
{[\%]}\end{array}$ & $\begin{array}{c}\operatorname{Err}_{\phi} \\
{[\%]}\end{array}$ \\
\hline 1 & 0.1122 & 9.65 & $\left(\begin{array}{cc}1 & \\
1.4913+i & 0.0854 \\
1.4886+i & 0.1089 \\
0.9918+i & 0.0782\end{array}\right)$ & 0.0258 & 0.0 & 0.0 & 0.003 \\
\hline 2 & 0.2814 & 6.02 & $\left.\begin{array}{cc}1 & \\
0.6110+i & 0.1691 \\
-0.6186-i & 0.0293 \\
-0.9734-i & 0.1504\end{array}\right)$ & 0.0963 & 0.0 & 0.17 & 0.012 \\
\hline 3 & 0.3897 & 5.54 & $\left.\begin{array}{cc}1 & \\
-0.3579+i & 0.1595 \\
-0.2663-i & 0.1711 \\
0.8770+i & 0.1354\end{array}\right)$ & 0.1815 & 0.0 & -0.89 & 3.90 \\
\hline 4 & 0.4302 & 3.21 & 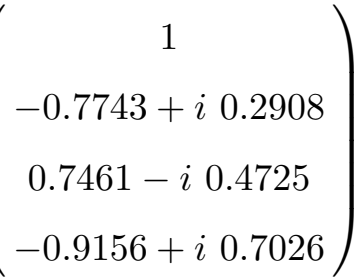 & 0.2828 & 0.14 & -1.53 & 0.07 \\
\hline
\end{tabular}

Table 5

Identified modal properties of system 1 . The corresponding exact values are given in Table 2 . 


\begin{tabular}{ccccccccccc}
\hline $\begin{array}{c}\text { Mode } \\
(\mathbf{j})\end{array}$ & $c_{t}, c_{f}$ & $\begin{array}{c}\bar{U}_{j} \\
{[\mathrm{~m}]}\end{array}$ & $\begin{array}{c}\tilde{f}_{j, \text { estim }} \\
{[\mathrm{Hz}]}\end{array}$ & $\begin{array}{c}\xi_{j, \text { estim }} \\
{[\%]}\end{array}$ & $Q_{\min }$ & $Q_{\max }$ & $Q_{\xi}$ & $Q$ & $t_{1}$ & $t_{\bar{U}_{j}}$ \\
\hline $\mathbf{1}$ & 5.0 & $2.0 * 10^{-3}$ & 0.11 & 10.0 & 2.5 & 16.46 & $\mathbf{7 . 0 4}$ & $\mathbf{7 . 0}$ & 50.58 & 67.91 \\
$\mathbf{2}$ & 5.0 & $2.0 * 10^{-3}$ & 0.28 & 6.0 & 6.36 & 41.9 & $\mathbf{1 1 . 7 6}$ & $\mathbf{1 1 . 7}$ & 33.26 & 41.86 \\
$\mathbf{3}$ & 4.0 & $2.0 * 10^{-3}$ & 0.39 & 6.0 & $\mathbf{1 9 . 5}$ & 72.95 & 11.76 & $\mathbf{1 9 . 5}$ & 31.86 & 33.37 \\
$\mathbf{4}$ & 4.0 & $2.0 * 10^{-3}$ & 0.43 & 3.3 & $\mathbf{2 1 . 5}$ & 80.42 & 21.4 & $\mathbf{2 1 . 5}$ & 31.86 & 46.05 \\
\hline
\end{tabular}

Table 6

Parameters related to the choice of $Q$-factor for the CWT analysis of system 1 with added white noise of standard deviation $\sigma_{n}=10^{-3} \mathrm{~m}$.

\begin{tabular}{|c|c|c|c|c|c|c|c|}
\hline $\begin{array}{l}\text { Mode } \\
\qquad(\mathbf{j})\end{array}$ & $\begin{array}{c}\tilde{f}_{j, \text { ident }} \\
{[\mathrm{Hz}]}\end{array}$ & $\begin{array}{c}\xi_{j, i d e n t} \\
{[\%]}\end{array}$ & $\phi_{j, \text { ident }}$ & $\tilde{I}_{n p, i d e n t}^{(j)}$ & $\begin{array}{c}\operatorname{Err}_{f} \\
{[\%]}\end{array}$ & $\begin{array}{c}\operatorname{Err}_{\xi} \\
{[\%]}\end{array}$ & $\begin{array}{c}E r r_{\phi} \\
{[\%]}\end{array}$ \\
\hline 1 & 0.1120 & 9.63 & $\left(\begin{array}{cc}1 & \\
1.4917+i & 0.0812 \\
1.4520+i & 0.0538 \\
0.9908+i & 0.0307\end{array}\right)$ & 0.0181 & -0.18 & -0.21 & 3.2 \\
\hline 2 & 0.2812 & 5.97 & $\begin{array}{c}1 \\
0.6197+i 0.1387 \\
-0.6350-i 0.0018 \\
-0.9384-i 0.1973\end{array}$ & 0.1056 & -0.07 & -0.66 & 4.4 \\
\hline 3 & 0.3882 & 5.03 & $\begin{array}{cc}1 & \\
-0.3481+i & 0.1842 \\
-0.2775-i & 0.1551 \\
0.8805+i & 0.0906\end{array}$ & 0.1885 & -0.33 & -10.02 & 3.7 \\
\hline 4 & 0.4297 & 3.25 & $\begin{array}{c}1 \\
-0.7662+i \quad 0.2677 \\
0.7129-i 0.4910 \\
-0.9268+i 0.7210\end{array}$ & 0.2934 & 0.02 & 0.31 & 2.6 \\
\hline
\end{tabular}

Table 7

Identified modal properties of system 1 with added white noise. The corresponding exact values are given in Table 2 . 


\begin{tabular}{|c|c|c|c|c|c|}
\hline $\begin{array}{l}\text { Mode } \\
\qquad(\mathbf{j})\end{array}$ & $\begin{array}{c}\tilde{f}_{j} \\
{[\mathrm{~Hz}]}\end{array}$ & $\begin{array}{c}\xi_{j} \\
{[\%]}\end{array}$ & $\phi_{j}$ & $I_{n p}^{(j)}$ & $\tilde{I}_{n p}^{(j)}$ \\
\hline 1 & 0.1077 & 37.07 & $\left(\begin{array}{cc}1 & \\
1.5293-i & 0.0994 \\
1.4232+i & 0.1496 \\
0.9204+i & 0.1577\end{array}\right)$ & 0.0965 & 0.0896 \\
\hline 2 & 0.2774 & 9.31 & $\begin{array}{c}1 \\
0.6576-i 0.1313 \\
-0.5596+i 0.4232 \\
-0.9420+i \quad 0.4554\end{array}$ & 0.2425 & 0.2494 \\
\hline 3 & 0.3906 & 4.11 & $\begin{array}{c}1 \\
-0.3408-i 0.0834 \\
-0.2112-i 0.2662 \\
0.7651+i 0.5939\end{array}$ & 0.3321 & 0.3483 \\
\hline 4 & 0.4211 & 6.26 & $\begin{array}{c}1 \\
-0.6627-i 0.2043 \\
0.6684-i 0.3004 \\
-0.7935+i \quad 0.6979\end{array}$ & 0.3828 & 0.3858 \\
\hline
\end{tabular}

Table 8

Modal properties of system 2, defined by the mass and stiffness matrices of Eq. (61) and the damping matrix (62), with $C_{11}=0.1$ and $C_{22}=2.2$.

\begin{tabular}{ccccccccccc}
\hline $\begin{array}{c}\text { Mode } \\
(\mathbf{j})\end{array}$ & $c_{t}, c_{f}$ & $\begin{array}{c}\bar{U}_{j} \\
{[\mathrm{~m}]}\end{array}$ & $\begin{array}{c}\tilde{f}_{j, \text { estim }} \\
{[\mathrm{Hz}]}\end{array}$ & $\begin{array}{c}\xi_{j, \text { estim }} \\
{[\%]}\end{array}$ & $Q_{\min }$ & $Q_{\max }$ & $Q_{\xi}$ & $Q$ & $t_{1}$ & $t_{\bar{U}_{j}}$ \\
\hline $\mathbf{1}$ & 5.5 & $10^{-5}$ & 0.11 & 37.0 & $\mathbf{2 . 7 5}$ & 14.96 & 1.75 & $\mathbf{2 . 7 5}$ & 21.43 & 43.02 \\
$\mathbf{2}$ & 5.5 & $10^{-5}$ & 0.28 & 9.0 & 7.0 & 38.09 & $\mathbf{7 . 8 2}$ & $\mathbf{7 . 8}$ & 24.37 & 62.09 \\
$\mathbf{3}$ & 5.5 & $10^{-5}$ & 0.39 & 4.0 & $\mathbf{3 5 . 7 5}$ & 53.05 & 17.66 & $\mathbf{3 5 . 7 5}$ & 80.23 & 106.74 \\
$\mathbf{4}$ & $5.8,5.4$ & $10^{-6}$ & 0.42 & 6.3 & $\mathbf{3 7 . 8}$ & 54.18 & 11.2 & $\mathbf{3 7 . 8}$ & 83.07 & 103.95 \\
\hline
\end{tabular}

Table 9

Parameters related to the choice of $Q$-factor for the CWT analysis of system 2. 


\begin{tabular}{ccccc}
\hline $\begin{array}{c}\text { Mode } \\
(\mathbf{j})\end{array}$ & $\Delta t_{u}$ & $\Delta t_{\psi_{b, a}}$ & $d f_{\text {estim }}$ & $c_{f} \frac{\Delta \omega_{\psi_{b, a}}}{2 \pi}=c_{f} \frac{\tilde{f}_{j, e s t i m}}{2 Q}$ \\
\hline $\mathbf{1}$ & 2.77 & 4.12 & $\mathbf{0 . 1 1}$ & $\mathbf{0 . 1 1}$ \\
$\mathbf{2}$ & $\mathbf{4 . 4 7}$ & $\mathbf{4 . 4 5}$ & 0.11 & 0.10 \\
$\mathbf{3}$ & 7.22 & 14.59 & $\mathbf{0 . 0 3}$ & $\mathbf{0 . 0 3}$ \\
$\mathbf{4}$ & 4.255 & 14.33 & $\mathbf{0 . 0 3}$ & $\mathbf{0 . 0 3}$ \\
\hline
\end{tabular}

Table 10

System 2. Characteristic time of the signal $\Delta t_{u}=\frac{1}{\sqrt{2}} \frac{1}{\xi_{j, e s t i m} 2 \pi \tilde{f}_{j, e s t i m}}$ and time standard deviation $\Delta t_{\psi_{b, a}}=\frac{1}{\sqrt{4 Q^{2}-2}} \frac{2 Q^{2}}{2 \pi \tilde{f}_{j, e s t i m}}$ of the translated and scaled mother wavelet (columns 1 and 2); estimated frequency distance $d f_{\text {estim }}$ between close peaks and frequency standard deviation $\Delta \omega_{\psi_{b, a}}$ multiplied by the factor $c_{f}$ (columns 3 and $4)$.

\begin{tabular}{|c|c|c|c|c|c|c|c|}
\hline $\begin{array}{l}\text { Mode } \\
\qquad(\mathrm{j})\end{array}$ & $\begin{array}{c}\tilde{f}_{j, \text { ident }} \\
{[\mathrm{Hz}]}\end{array}$ & $\begin{array}{c}\xi_{j, i d e n t} \\
{[\%]}\end{array}$ & $\phi_{j, i d e n t}$ & $\tilde{I}_{n p, i d e n t}^{j}$ & $\begin{array}{c}\operatorname{Err}_{f} \\
{[\%]}\end{array}$ & $\begin{array}{c}\operatorname{Err}_{\xi} \\
{[\%]}\end{array}$ & $\begin{array}{c}E r r_{\phi} \\
{[\%]}\end{array}$ \\
\hline 1 & 0.1076 & 37.33 & $\left(\begin{array}{cc}1 & \\
1.5297-i & 0.1156 \\
1.4179+i & 0.1657 \\
0.9137+i & 0.1736\end{array}\right)$ & 0.100 & -0.10 & 0.70 & 1.16 \\
\hline 2 & 0.2774 & 9.32 & 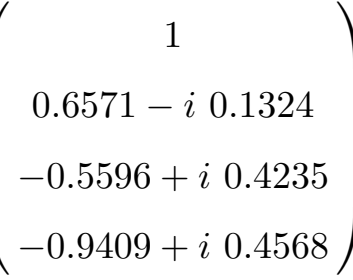 & 0.250 & 0.0 & 0.11 & 0.12 \\
\hline 3 & 0.3912 & 3.97 & $\begin{array}{c}1 \\
-0.3347-i 0.0955 \\
-0.1870-i 0.2854 \\
0.7291+i 0.6428\end{array}$ & 0.384 & 0.15 & -3.41 & 4.71 \\
\hline 4 & 0.4200 & 5.87 & $\begin{array}{c}1 \\
-0.6148-i 0.2684 \\
0.6599-i 0.3777 \\
-0.6461+i 0.9308\end{array}$ & 0.513 & -0.26 & -6.23 & 16.80 \\
\hline
\end{tabular}

Table 11

Identified modal properties of system 2 . The corresponding exact values are given in Table 8. 


\begin{tabular}{ccccccccccc}
\hline $\begin{array}{c}\text { Mode } \\
(\mathbf{j})\end{array}$ & $c_{t}, c_{f}$ & $\begin{array}{c}\bar{U}_{j} \\
{[\mathrm{~m}]}\end{array}$ & $\begin{array}{c}\tilde{f}_{j, \text { estim }} \\
{[\mathrm{Hz}]}\end{array}$ & $\begin{array}{c}\xi_{j, \text { estim }} \\
{[\%]}\end{array}$ & $Q_{\min }$ & $Q_{\max }$ & $Q_{\xi}$ & $Q$ & $t_{1}$ & $t_{\bar{U}_{j}}$ \\
\hline $\mathbf{1}$ & 5.0 & $2.0 * 10^{-3}$ & 0.11 & 37.0 & $\mathbf{2 . 5}$ & 16.46 & 1.75 & $\mathbf{2 . 5}$ & 20.81 & 23.02 \\
$\mathbf{2}$ & 5.5 & $2.0 * 10^{-3}$ & 0.28 & 9.0 & 7.0 & 38.1 & $\mathbf{7 . 8 2}$ & $\mathbf{7 . 8}$ & 24.42 & 29.53 \\
$\mathbf{3}$ & 4.0 & $2.0 * 10^{-3}$ & 0.39 & 4.0 & $\mathbf{2 6 . 0}$ & 72.9 & 17.66 & $\mathbf{2 6}$ & 42.44 & 53.48 \\
$\mathbf{4}$ & & & & & & & & & & \\
\end{tabular}

Table 12

Parameters related to the choice of $Q$-factor for the CWT analysis of system 1 with added white noise of standard deviation $\sigma_{n}=10^{-3}$.

\begin{tabular}{|c|c|c|c|c|c|c|c|}
\hline $\begin{array}{l}\text { Mode } \\
\qquad(\mathrm{j})\end{array}$ & $\begin{array}{c}\tilde{f}_{j, i d e n t} \\
{[\mathrm{~Hz}]}\end{array}$ & $\begin{array}{c}\xi_{j, \text { ident }} \\
{[\%]}\end{array}$ & $\phi_{j, i d e n t}$ & $\tilde{I}_{n p, i d e n t}^{(j)}$ & $\begin{array}{c}\operatorname{Err}_{f} \\
{[\%]}\end{array}$ & $\begin{array}{c}\operatorname{Err}_{\xi} \\
{[\%]}\end{array}$ & $\begin{array}{c}E r r_{\phi} \\
{[\%]}\end{array}$ \\
\hline 1 & 0.1082 & 41.9 & $\left(\begin{array}{cc}1 & \\
1.5044-i & 0.2688 \\
1.3564+i & 0.2175 \\
0.8664-i & 0.1566\end{array}\right)$ & 0.149 & 0.46 & 12.9 & 14.9 \\
\hline 2 & 0.2772 & 9.2 & $\left.\begin{array}{c}1 \\
0.6431-i 0.0493 \\
-0.6075+i \quad 0.3934 \\
-0.9500+i \quad 0.4227\end{array}\right)$ & 0.236 & -0.1 & -1.1 & 6.1 \\
\hline 3 & 0.3906 & 3.8 & $\left.\begin{array}{c}1 \\
-0.1139-i 0.2646 \\
-0.1427-i 0.3220 \\
0.6292+i 0.7398\end{array}\right)$ & 0.49 & 0.0 & -7.8 & 24.7 \\
\hline
\end{tabular}

Table 13

Identified modal properties of system 2 with added white noise. The corresponding exact values are given in Table 8 . 


\begin{tabular}{cccccccccccc}
\hline $\begin{array}{c}\text { Mode } \\
(\mathbf{j})\end{array}$ & Type & $c_{t}, c_{f}$ & $\begin{array}{c}\bar{U}_{j} \\
{[\mathrm{~m}]}\end{array}$ & $\begin{array}{c}\tilde{f}_{j, \text { estim }} \\
{[\mathrm{Hz}]}\end{array}$ & $\begin{array}{c}\xi_{j, \text { estim }} \\
{[\%]}\end{array}$ & $Q_{\min }$ & $Q_{\max }$ & $Q_{\xi}$ & $Q$ & $t_{1}$ & $t_{\bar{U}_{j}}$ \\
\hline $\mathbf{1}$ & $(1,1)$ & 4.0 & $6.3 * 10^{-6}$ & 27 & 3.0 & 6.75 & 49.70 & $\mathbf{2 3 . 5 6}$ & $\mathbf{2 3 . 5}$ & 0.552 & 0.613 \\
$\mathbf{2}$ & $(0,2)$ & 4.0 & $4.0 * 10^{-6}$ & 35 & 3.0 & 8.75 & 64.43 & $\mathbf{2 3 . 5 6}$ & $\mathbf{2 3 . 5}$ & 0.431 & 0.460 \\
$\mathbf{3}$ & $(2,0)$ & 4.0 & $2.5 * 10^{-6}$ & 58 & 3.0 & 14.50 & 106.76 & $\mathbf{2 3 . 5 6}$ & $\mathbf{2 3 . 5}$ & 0.264 & 0.310 \\
$\mathbf{4}$ & $(1,2)$ & 4.0 & $2.5 * 10^{-6}$ & 66 & 3.0 & 16.51 & 121.50 & $\mathbf{2 3 . 5 6}$ & $\mathbf{2 3 . 5}$ & 0.233 & 0.258 \\
$\mathbf{5}$ & $(2,1)$ & 4.0 & $2.5 * 10^{-6}$ & 77.5 & 3.0 & 13.47 & 142.66 & $\mathbf{2 3 . 5 6}$ & $\mathbf{2 3 . 5}$ & 0.188 & 0.221 \\
$\mathbf{6}$ & $(0,3)$ & 4.0 & $1.3 * 10^{-6}$ & 107 & 3.0 & 7.64 & 196.96 & $\mathbf{2 3 . 5 6}$ & $\mathbf{2 3 . 5}$ & 0.140 & 0.164 \\
$\mathbf{7}$ & $(1,3)$ & 4.0 & $3.6 * 10^{-6}$ & 135 & 3.0 & 10.8 & 289.92 & $\mathbf{2 3 . 5 6}$ & $\mathbf{2 0}$ & 0.094 & 0.108 \\
$\mathbf{8}$ & $(2,2)$ & 4.0 & $1.3 * 10^{-6}$ & 135 & 3.0 & 10.38 & 248.50 & $\mathbf{2 3 . 5 6}$ & $\mathbf{2 3 . 5}$ & 0.111 & 0.135 \\
$\mathbf{9}$ & $(3,0)$ & 4.0 & $5.0 * 10^{-7}$ & 161 & 2.0 & 14.64 & 296.12 & $\mathbf{3 5 . 3 5}$ & $\mathbf{3 5}$ & 0.139 & 0.160 \\
$\mathbf{1 0}$ & $(3,1)$ & 4.0 & $5.0 * 10^{-7}$ & 182 & 2.5 & 17.33 & 335.02 & $\mathbf{2 8 . 2 8}$ & $\mathbf{2 8}$ & 0.100 & 0.131 \\
\hline Table 14 & & & & & & & & & & &
\end{tabular}

Parameters related to the choice of $Q$-factor for the CWT analysis of the plexiglass plate.

\begin{tabular}{ccccccccc}
\hline & \multicolumn{3}{c}{$F E M$} & \multicolumn{3}{c}{ DATA SET 1 } & \multicolumn{3}{c}{ DATA SET 2 } \\
Mode & Type & $f_{j}$ & $\tilde{f}_{j, \text { ident }}$ & $\xi_{j, \text { ident }}$ & $\tilde{I}_{n p, \text { ident }}^{(j)}$ & $\tilde{f}_{j, \text { ident }}$ & $\xi_{j, \text { ident }}$ & $\tilde{I}_{n p, \text { ident }}^{(j)}$ \\
$(\mathbf{j})$ & & {$[\mathrm{Hz}]$} & {$[\mathrm{Hz}]$} & {$[\%]$} & & {$[\mathrm{Hz}]$} & {$[\%]$} & \\
\hline $\mathbf{1}$ & $(1,1)$ & 26.88 & 26.7 & 2.7 & 0.072 & & \\
$\mathbf{2}$ & $(0,2)$ & 35.26 & 35.1 & 3.0 & 0.309 & & \\
$\mathbf{3}$ & $(2,0)$ & 59.53 & 58.6 & 2.8 & 0.049 & & \\
$\mathbf{4}$ & $(1,2)$ & 65.58 & 66.6 & 3.1 & 0.042 & & & \\
$\mathbf{5}$ & $(2,1)$ & 75.92 & 78.0 & 3.05 & 0.077 & & & \\
$\mathbf{6}$ & $(0,3)$ & 107.08 & 107.0 & 2.95 & 0.175 & & & \\
$\mathbf{7}$ & $(1,3)$ & 128.02 & - & - & - & 133.2 & 1.80 & 0.471 \\
$\mathbf{8}$ & $(2,2)$ & 129.55 & 134.2 & 2.9 & 0.337 & - & - & - \\
$\mathbf{9}$ & $(3,0)$ & 158.57 & 161.1 & 2.3 & 0.147 & 161.3 & 2.1 & 0.146 \\
$\mathbf{1 0}$ & $(3,1)$ & 179.46 & 182.2 & 2.25 & 0.105 & 182.4 & 2.45 & 0.109 \\
\hline
\end{tabular}

Table 15

Modal frequencies of the undamped finite element model of the plexiglass plate and the corresponding identified modal frequencies, damping ratios and nonproportionality indices. 